\title{
Stability Analysis of a Repairable System with Warning Device and Repairman Vacation
}

\author{
Yangli Ren, Lina Guo, and Lingling Zhang \\ Department of Mathematics, Taiyuan University of Technology, Taiyuan 030024, China \\ Correspondence should be addressed to Lina Guo; guolina982@163.com
}

Received 19 April 2013; Accepted 7 August 2013

Academic Editor: Gen-Qi Xu

Copyright (c) 2013 Yangli Ren et al. This is an open access article distributed under the Creative Commons Attribution License, which permits unrestricted use, distribution, and reproduction in any medium, provided the original work is properly cited.

\begin{abstract}
This paper considers a simple repairable system with a warning device and a repairman who can have delayed-multiple vacations. By Markov renewal process theory and the probability analysis method, the system is first transformed into a group of integrodifferential equations. Then, the existence and uniqueness as well as regularity of the system dynamic solution are discussed with the functional analysis method. Further, the asymptotic stability, especially the exponential stability of the system dynamic solution, is studied by using the strongly continuous semigroup theory or $C_{0}$ semigroup theory. The reliability indices and some applications (such as the comparisons of indices and profit of systems with and without warning device), as well as numerical examples, are presented at the end of the paper.
\end{abstract}

\section{Introduction}

A repairable system is a system which, after failing to perform one or more of its functions satisfactorily, can be restored to fully satisfactory performance by any method, rather than the replacement of the entire system. With different repair levels, repair can be broken down into three categories (see [1]): perfect repair, normal repair, and minimal repair. A perfect repair can restore a system to an "as good as new" state, a normal repair is assumed to bring the system to any condition, and a minimal repair, or imperfect repair, can restore the system to the exact state it was before failure.

Repairable system is not only a kind of important system discussed in reliability theory but also one of the main objects studied in reliability mathematics. Since the 1960s, various repairable system models have been established and researched.

However, in traditional repairable systems, it is assumed that the repairman or server remains idle until a failed component presents. But as Mobley [2] pointed out, onethird of all maintenance costs were wasted as the result of unnecessary or improper maintenance activities. Today, the role of maintenance tends to be a "profit contributor." Therefore, much more profit can be produced when the repairman in a system might take a sequence of vacations in the idle time.
Repairman's vacation may literally mean a lack of work or repairman taking another assigned job. From the perspective of rational use of human resources, the introduction of repairman's vacation makes modeling of the repairable system more realistic and flexible. This is due to the fact that in practice, the vast majority of small-and medium-sized enterprises (SMEs) cannot afford to hire a full-time repairman. So, the repairman in SMEs usually plays two roles: one for looking after the equipment and one for other duties. Under normal circumstances, the repairman has to periodically check the status of the system. If he finds that the system failed, he repairs it immediately after the end of vacation; otherwise, he will leave the system for other duties or for a vacation.

Vacation model originally arised in queueing theory and has been well studied in the past three decades and successfully applied in many areas such as manufacturing/service and computer/communication network systems. Excellent surveys on the earlier works of vacation models have been reported by Doshi [3], Takagi [4], and Tian and Zhang [5]. A number of works (e.g., please see [6-10] and references therein) have recently appeared in the queueing literature in which concepts of different control operating politics along with vacations have been discussed. And Ke et al. [11] provided a summary of the most recent research works on 
vacation queueing systems in the past 10 years, in which a wide class of vacation policies for governing the vacation mechanism is presented.

In the past decade, inspired by the vacation queueing theory, some researchers introduced vacation model into repairable systems. The available references concerning repairman vacation in repairable systems can be classified into two categories: one is focused on the system indices and the other is the optimization problems.

For the first category, Jain and Rakhee [12] considered the bilevel control policy for a machining system having two repairmen. One turns on when queue size of failed units reaches a preassigned level. The other's provision in case of long queue of failed units may be helpful in reducing the backlog. The steady state queue size distribution is obtained by applying the recursive method. $\mathrm{Hu}$ et al. [13] studied the steady-state availability and the mean up-time of a seriesparallel repairable system consisting of one master control unit, two slave units, and a single repairman who operates single vacation by using the supplementary variable method and the vector Markov process theory. Q. T. Wu and S. M. Wu [14] analyzed some reliability indices of a cold standby system consisting of two repairable units, a switch and a repairman who may not always be at the job site or take vacation. Yuan [15] and Yuan and Cui [16] studied a k-out-of-n:G system and a consecutive-k-out-of-n:F system, respectively, with $\mathrm{R}$ repairmen who can take multiple vacations and by using Markov model; the analytical solution of some reliability indices was discussed. Yuan and $\mathrm{Xu}$ [17] studied a deteriorating system with a repairman who can have multiple vacations. By means of the geometric process and the supplementary variable techniques, a group of partial differential equations of the system was presented, and some reliability indices were derived. Ke and Wu [18] studied a multiserver machine repair model with standbys and synchronous multiple vacations, and the stationary probability vectors were obtained by using the matrix-analytical approach and the technique of matrix recursive.

For the second category, Ke and Wang [19] studied a machine repair problem consisting of $\mathrm{M}$ operating machines with two types of spare machines and R servers (repairmen) who can take different vacation policies. The steady-state probabilities of the number of failed machines in the system as well as the performance measures were derived by using the matrix geometric theory, and a direct search algorithm was used to determine the optimal values of the number of two types of spares and the number of servers while maintaining a minimum specified level of system availability. Jia and Wu [20] considered a replacement policy for a repairable system that can not be repaired "as good as new" with a repairman who can have multiple vacations. By using geometric processes, the explicit expression of the expected cost rate was derived, and the corresponding optimal policy was determined analytically and numerically. Yuan and $\mathrm{Xu}[21,22]$ considered, respectively, a deteriorating repairable system and a cold standby repairable system with two different components of different priority in use, both with one repairman who can take multiple vacations. The explicit expression of the expected cost rate was given, and an optimal replacement policy was discussed. Yu et al. [23] analyzed a phase-type geometric process repair model with spare device procurement lead time and repairman's multiple vacations. Employing the theory of renewal reward process, the explicit expression of the long-run average profit rate for the system was derived, and the optimal maintenance policy was also numerically determined.

However, to the best knowledge of the authors, whichever the catalogue, the references above only concentrated on the steady state (the steady-state indices or the steady-state optimization problems) of the systems. It is because that the transient behavior of a system is difficult to be studied. Therefore, in reliability study researchers usually substitute the steady-state solution for the instantaneous one of a system, for the steady-state solution can be easily obtained by Laplace transform and a limit theorem. Whereas, Laplace transform should be based on the two hypotheses: (1) the instantaneous solution of the interested system existed and (2) the instantaneous solution of the system is stable. Whether the hypotheses hold or not is still an open question and should be justified. Moreover, the substitution of the steadystate solution for the instantaneous one is not always rational. For detailed information or explanations, please see [24, 25].

Warning systems emerge in the background of repairable systems which are stepping into the times of requiring of both advanced warning and real-time fault detection. The socalled warning system is able to send emergency signals and report dangerous situations prior to disasters, catastrophes and/or other dangers need to watch out based on previous experiences and/or observed possible omens. Real-time warning systems play an important role in fault management in banking, telecommunications, securities, electric power, and other industries. If the warning prompts during system operation, operating staff can choose shut down the system, operate carefully, or repair the system. Warning systems can help users to achieve the 24-hour uninterrupted real-time monitoring and alerting during running of various types of network infrastructure sand application services. Therefore, there is a need to study the repairable systems with warning device.

This paper considers a simple repairable system with a warning device and a repairman who can have delayedmultiple vacations. The delayed-multiple vacations mean that the repairman will not leave for a vacation immediately if there is no component failed. However, there is a stochastic vacation-preparing period in which if a failed component appears he will stop the vacation preparing and serve it immediately; otherwise, he will take a rest on the end of the vacation-preparing period. When he returns from a vacation, he will either deal with the failed components waiting in the system or prepare for another vacation. In this paper, we are devoted to studying the asymptotic behavior of the system by strongly continuous semigroup theory and make comparisons of indices (such as reliability, availability, and the probability of the repairman's vacation) and profit of the two systems with and without warning device.

The paper is structured as follows. The coming section introduces the system model specifically and expresses it into a group of integrodifferential equations by Markov 
renewal process theory and the probability analysis method. Section 3 discusses the existence and uniqueness as well as the regularity of the system dynamic solution by the functional analysis method. Section 4 studies the asymptotic behavior of the system by strongly continuous semigroup theory or $C_{0}$ semigroup theory. Section 5 presents some reliability indices of the system, and the steady-state indices are discussed from the viewpoint of eigenfunction of the system operator. In Section 6, comparisons of indices and profit of systems with and without warning device are made. And a brief conclusion is offered in the last section.

\section{System Formulation}

The system model of interest is a simple repairable system (i.e., a repairable system with a unit and a repairman) with repairman vacation and a warning device. It is described specifically as follows: at the initial time $t=0$, the unit is new, the system begins to work, and the repairman starts to prepare for the vacation. If the unit fails in the delayedvacation period, the repairman deals with it immediately, and the delayed vacation is terminated. Otherwise, he leaves for a vacation after the delayed-vacation period ends. If the warning device sends alerts in the delayed-vacation period, the repairman will stay in the system until the unit fails. Whenever the repairman returns from a vacation, he either prepares for the next vacation if the unit is working or deals with the failed unit immediately or stays in the system if the warning device has sent alerts. The repair facility neither failed nor deteriorated. The unit is repaired as good as new. Further, we assume the following.

(1) The distribution function of the working time of the unit is $F(t)=1-e^{-\lambda t}, t \geq 0, \lambda$ is a positive constant, and the distribution function of its repair time is $G(t)=\int_{0}^{t} g(x) \mathrm{d} x=1-e^{-\int_{0}^{t} \mu(x) \mathrm{d} x}$ and $\int_{0}^{\infty} t \mathrm{~d} G(t)=$ $1 / b$.

(2) The distribution function of the delayed-vacation time of the repairman is $D(t)=1-e^{-\varepsilon t}, t \geq 0, \varepsilon$ is a positive constant, and the distribution function of his vacation time is $V(t)=1-e^{-\mu_{0} t}, \mu_{0}$ is a positive constant.

(3) The distribution function of the time of the warning device from its beginning to work to its first sending alerts is $U(t)=1-e^{-\alpha_{0} t}, t \geq 0 ; \alpha_{0}$ is a positive constant.

(4) The above stochastic variables are independent of each other.

Set $N(t)$ to be the state in which the system is at time $t$, and assume all the possible states as follows:

0 : the system is working, and the repairman is preparing for the vacation;

1: the system is working, and the repairman is on vacation;

2: the system is warning, and the repairman is in the system;
3: the system is warning, and the repairman is on vacation;

4: the unit failed, and the repairman is on vacation;

5: the repairman is dealing with the failed unit.

Then, by using probability analysis method, the system model can be described as the following group of integrodifferential equations:

$$
\begin{gathered}
\left(\frac{\mathrm{d}}{\mathrm{d} t}+\varepsilon+\alpha_{0}\right) P_{0}(t)=\mu_{0} P_{1}(t)+\int_{0}^{\infty} \mu(x) P_{5}(t, x) \mathrm{d} x \\
\left(\frac{\mathrm{d}}{\mathrm{d} t}+\mu_{0}+\alpha_{0}\right) P_{1}(t)=\varepsilon P_{0}(t) \\
\left(\frac{\mathrm{d}}{\mathrm{d} t}+\lambda\right) P_{2}(t)=\alpha_{0} P_{0}(t)+\mu_{0} P_{3}(t), \\
\left(\frac{\mathrm{d}}{\mathrm{d} t}+\mu_{0}+\lambda\right) P_{3}(t)=\alpha_{0} P_{1}(t) \\
\left(\frac{\mathrm{d}}{\mathrm{d} t}+\mu_{0}\right) P_{4}(t)=\lambda P_{3}(t) \\
{\left[\frac{\partial}{\partial t}+\frac{\partial}{\partial x}+\mu(x)\right] P_{5}(t, x)=0 .}
\end{gathered}
$$

The boundary condition is

$$
P_{5}(t, 0)=\lambda P_{2}(t)+\mu_{0} P_{4}(t) .
$$

The initial conditions are

$$
P_{0}(0)=1 \text {, the others equal to } 0 \text {. }
$$

Here, $P_{i}(t)$ represents the probability that the system is in state $i$ at time $t, i=0,1, \ldots, 4$, and $P_{5}(t, x) \mathrm{d} x$ represents the probability that the system is in state 5 with elapsed repair time lying in $[x, x+\mathrm{d} x)$ at time $t$.

Concerning the practical background, we can assume that

$$
\mu(x) \geq 0, \quad \mu=\sup _{x \in[0, \infty)} \mu(x)<\infty
$$

\section{Existence and Uniqueness of System Solution}

In this section, we will study the existence and uniqueness as well as the regularity of the system solution. Firstly, we will transform the system (1)-(3) into an equivalent integral problem $(\mathrm{P})$ by the method of characteristics. Secondly, the existence and uniqueness of the local solution of problem (P) are discussed by using the fixed point theory. Then, the existence and uniqueness of the global solution of problem (P) is further studied by a uniform priori estimate. Thus, the existence and uniqueness of the solution of system (1)-(3) are obtained. Moreover, the regularity or the $C^{1}$ continuity of the system solution is also discussed. 
3.1. Unique Existence of System Local Solution. For convenience, we will give some notations. Let

$$
L_{1}=L^{1}[0, \infty), \quad V_{0}=C[0, T], \quad V_{1}=C\left([0, T], L_{1}\right)
$$

with norm

$$
\begin{gathered}
\|\varphi\|_{L_{1}}=\int_{0}^{\infty}|\varphi(x)| \mathrm{d} x, \\
\|f\|_{V_{0}}=\max _{t \in[0, T]}|f(t)|, \\
\|g\|_{V_{1}}=\max _{t \in[0, T]} \int_{0}^{\infty}|g(t, x)| \mathrm{d} x .
\end{gathered}
$$

Choose

$$
M=\left\{q \in V_{0} \mid q(0)=1, q \geq 0,\|q\| \leq 2\right\} .
$$

Clearly, $M$ is a closed subspace of $V_{0}$.

By the method of characteristics [26], the following equivalent proposition can be easily obtained [27, 28].

Theorem 1. For a given constant $T>0, P_{i}(t) \in V_{0}, i=$ $0,1, \ldots, 4, P_{5}(t, x) \in V_{1}$ are the solution to (1)-(3) if and only if they are the solution to the following integral problem $(P)$ :

$$
\begin{gathered}
P_{0}(t)=e^{-\left(\varepsilon+\alpha_{0}\right) t}+\int_{0}^{t}\left[\mu_{0} P_{1}(s)+\int_{0}^{\infty} \mu(x) P_{5}(s, x) \mathrm{d} x\right] \\
\times e^{-\left(\varepsilon+\alpha_{0}\right)(t-s)} \mathrm{d} s, \\
P_{1}(t)=\int_{0}^{t} \varepsilon P_{0}(s) e^{-\left(\mu_{0}+\alpha_{0}\right)(t-s)} \mathrm{d} s, \\
P_{2}(t)=\int_{0}^{t}\left[\alpha_{0} P_{0}(s)+\mu_{0} P_{3}(s)\right] e^{-\lambda(t-s)} \mathrm{d} s, \\
P_{3}(t)=\int_{0}^{t} \alpha_{0} P_{1}(s) e^{-\left(\mu_{0}+\lambda\right)(t-s)} \mathrm{d} s, \\
P_{4}(t)=\int_{0}^{t} \lambda P_{3}(s) e^{-\mu_{0}(t-s)} \mathrm{d} s,
\end{gathered}
$$$$
P_{5}(t, x)= \begin{cases}0, & x \geq t \\ {\left[\lambda P_{2}(t-x)+\mu_{0} P_{4}(t-x)\right] e^{-\int_{0}^{x} \mu(\tau) \mathrm{d} \tau},} & x<t .\end{cases}
$$

Clearly, to get the existence and uniqueness of the solution of system (1)-(3), it is necessary to study the existence and uniqueness of the solution of the above integral problem (P). To this end, for any $q \in V_{0}$, we define six operators as follows:

$$
\begin{aligned}
K_{0}(q)(t)= & e^{-\left(\varepsilon+\alpha_{0}\right) t} \\
& +\int_{0}^{t}\left[\mu_{0} K_{1}(q)(s)\right. \\
& \left.\quad+\int_{0}^{\infty} \mu(x) K_{5}(q)(s, x) \mathrm{d} x\right] \\
& \times e^{-\left(\varepsilon+\alpha_{0}\right)(t-s)} \mathrm{d} s
\end{aligned}
$$

$$
K_{1}(q)(t)=\int_{0}^{t} \varepsilon q(s) e^{-\left(\mu_{0}+\alpha_{0}\right)(t-s)} \mathrm{d} s
$$

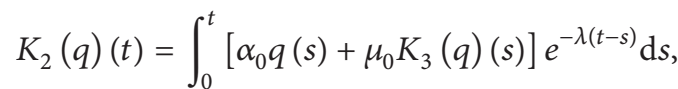

$$
\begin{aligned}
K_{5}(q)(t, x)= & {\left[\lambda K_{2}(q)(t-x)+\mu_{0} K_{4}(q)(t-x)\right] } \\
& \times e^{-\int_{0}^{x} \mu(\tau) \mathrm{d} \tau} .
\end{aligned}
$$

It can be seen that for $q \in V_{0}$, if the operators $K_{i}, i=1,2, \ldots, 5$ are determined, it needs only to get the fixed point of the operator $K_{0}$ in order to get the existence and uniqueness of the solution of the integral problem $(\mathrm{P})$.

From (9)-(13), the following two lemmas can be easily obtained.

Lemma 2. For a given constant $T>0$ such that $t \in[0, T]$, then for any $q \in M$, there exist unique and nonnegative $K_{i}(q) \in V_{0}$, $i=1, \ldots, 4$ and $K_{5}(q) \in V_{1}$ satisfying $(9)-(13)$.

Lemma 3. For a given constant $T>0$ such that $t \in[0, T]$, then for any $q, \tilde{q} \in M$, the following estimations hold:

$$
\begin{gathered}
\left\|K_{1}(q)\right\| \leq 2 \varepsilon T, \\
\left\|K_{1}(q)-K_{1}(\tilde{q})\right\| \leq \varepsilon T\|q-\tilde{q}\|, \\
\left\|K_{3}(q)\right\| \leq 2 \alpha_{0} \varepsilon T^{2}, \\
\left\|K_{3}(q)-K_{3}(\tilde{q})\right\| \leq \alpha_{0} \varepsilon T^{2}\|q-\tilde{q}\|, \\
\left\|K_{2}(q)\right\| \leq 2 \alpha_{0} T\left(1+\mu_{0} \varepsilon T^{2}\right), \\
\left\|K_{2}(q)-K_{2}(\tilde{q})\right\| \leq \alpha_{0} T\left(1+\mu_{0} \varepsilon T^{2}\right)\|q-\tilde{q}\|, \\
\left\|K_{4}(q)\right\| \leq 2 \lambda \alpha_{0} \varepsilon T^{3}, \\
\left\|K_{4}(q)-K_{4}(\tilde{q})\right\| \leq \lambda \alpha_{0} \varepsilon T^{3}\|q-\tilde{q}\|, \\
\left\|K_{5}(q)\right\| \leq 2 \lambda \alpha_{0} T^{2}\left(1+2 \mu_{0} \varepsilon T^{2}\right), \\
\left\|K_{5}(q)-K_{5}(\tilde{q})\right\| \leq \lambda \alpha_{0} T^{2}\left(1+2 \mu_{0} \varepsilon T^{2}\right)\|q-\tilde{q}\| .
\end{gathered}
$$

Theorem 4. There exists a $T=T_{0}>0$, such that $K_{0}$ has a unique fixed point on $M$.

Proof. We prove the theorem in two steps. Firstly, we prove that the operator $K_{0}$ is a mapping from $M$ to $M$. From the definition of $K_{0}$, we can know that if $q \in M$, then $K_{0}(q) \in V_{0}$ and $K_{0}(q)(0)=1$. Choose $0<T_{0}<1$ satisfying

$$
T_{0}^{2}\left[\mu_{0} \varepsilon+\mu \lambda \alpha_{0}\left(1+2 \mu_{0} \varepsilon\right)\right]<\frac{1}{2} .
$$


Then, from (8) and Lemmas 2 and 3, it can be derived that

$$
\begin{aligned}
\left\|K_{0}(q)\right\|= & \max _{t \in[0, T]}\left|K_{0}(q)(t)\right| \leq 1+2 T^{2} \\
& \times\left[\mu_{0} \varepsilon+\mu \lambda \alpha_{0} T\left(1+2 \mu_{0} \varepsilon T^{2}\right)\right] \\
< & 1+2 T^{2}\left[\mu_{0} \varepsilon+\mu \lambda \alpha_{0}\left(1+2 \mu_{0} \varepsilon\right)\right]<2 .
\end{aligned}
$$

This implies that $K_{0}(q) \in M$.

Secondly, we prove that the operator $K_{0}$ is a strictly compressed mapping on $M$. For any $q, \tilde{q} \in M$, from (8) and Lemma 3, we have

$$
\begin{aligned}
\left\|K_{0}(q)-K_{0}(\tilde{q})\right\| \\
\quad \leq T^{2}\left[\mu_{0} \varepsilon+\mu \lambda \alpha_{0} T\left(1+2 \mu_{0} \varepsilon T^{2}\right)\right]\|q-\tilde{q}\| \\
\quad<T^{2}\left[\mu_{0} \varepsilon+\mu \lambda \alpha_{0}\left(1+2 \mu_{0} \varepsilon\right)\right]\|q-\tilde{q}\| \\
\quad<\frac{1}{2}\|q-\tilde{q}\| .
\end{aligned}
$$

This means that $K_{0}$ is strictly compressed. According to the Banach contraction mapping principle combining the above two steps, it can be deduced readily that $K_{0}$ has a unique fixed point on $M$. The proof of Theorem 4 is completed.

Theorems 1 and 4 combing Lemma 2 follows the existence and uniqueness of the local solution of system (1)-(3).

Theorem 5 (existence and uniqueness of local solution). There exists a $T=T_{0}>0$ such that the system (1)-(3) has a unique nonnegative local solution $\left(P_{0}, P_{1}, \ldots, P_{4}, P_{5}\right) \in V_{0}^{5} \times V_{1}$.

3.2. Unique Existence of System Global Solution. In this section, we will prove the existence and uniqueness of the global solution of system (1)-(3) by a uniform priori estimate and extension theorem.

Lemma 6. For a given constant $T>0$, if $\left(P_{0}, P_{1}, \ldots, P_{4}, P_{5}\right) \in$ $V_{0}^{5} \times V_{1}$ is the nonnegative solution of system (1)-(3), then one has:

$$
E(t) \leq e^{Q T}, \quad \forall t \in[0, T]
$$

where $E(t)=\sum_{i=0}^{4} P_{i}(t)+\int_{0}^{\infty} P_{5}(t, x) \mathrm{d} x, Q=\max \left\{\varepsilon+\alpha_{0}, \mu_{0}+\right.$ $\left.\alpha_{0}, \mu_{0}+\lambda, \mu\right\}$ and $\mu$ is defined in (4).

Proof. Because the solution of system (1)-(3) is the solution of problem $(\mathrm{P})$, the estimation of the system solution can be obtained easily as follows:

$$
\begin{aligned}
P_{0}(t) \leq & e^{-\left(\varepsilon+\alpha_{0}\right) t}+\int_{0}^{t} \mu_{0} P_{1}(s) \mathrm{d} s \\
& +\int_{0}^{t}\left[\int_{0}^{\infty} \mu(x) P_{5}(s, x) \mathrm{d} x\right] \mathrm{d} s
\end{aligned}
$$

$$
\begin{gathered}
\leq 1+\mu_{0} \int_{0}^{t} P_{1}(s) \mathrm{d} s \\
+\mu \int_{0}^{t}\left[\int_{0}^{\infty} P_{5}(s, x) \mathrm{d} x\right] \mathrm{d} s \\
P_{1}(t) \leq \varepsilon \int_{0}^{t} P_{0}(s) \mathrm{d} s \\
P_{2}(t) \leq \alpha_{0} \int_{0}^{t} P_{0}(s) \mathrm{d} s+\mu_{0} \int_{0}^{t} P_{3}(s) \mathrm{d} s, \\
P_{3}(t) \leq \alpha_{0} \int_{0}^{t} P_{1}(s) \mathrm{d} s, \\
\int_{0}^{\infty} P_{4}(t) \leq \lambda \int_{0}^{t} P_{3}(s) \mathrm{d} s, \\
+\mu_{0} \int_{0}^{\infty} P_{4}(t-x) \mathrm{d} x \\
=\lambda \int_{0}^{t} P_{2}(s) \mathrm{d} s+\mu_{0}(t-x) \mathrm{d} x
\end{gathered}
$$

Thus,

$$
\begin{aligned}
E(t)= & \sum_{i=0}^{4} P_{i}(t)+\int_{0}^{\infty} P_{5}(t, x) \mathrm{d} x \\
\leq & 1+\left(\varepsilon+\alpha_{0}\right) \int_{0}^{t} P_{0}(s) \mathrm{d} s \\
& +\left(\mu_{0}+\alpha_{0}\right) \int_{0}^{t} P_{1}(s) \mathrm{d} s+\lambda \int_{0}^{t} P_{2}(s) \mathrm{d} s \\
& +\left(\mu_{0}+\lambda\right) \int_{0}^{t} P_{3}(s) \mathrm{d} s+\mu_{0} \int_{0}^{t} P_{4}(s) \mathrm{d} s \\
& +\mu \int_{0}^{t}\left[\int_{0}^{\infty} P_{5}(s, x) \mathrm{d} x\right] \mathrm{d} s .
\end{aligned}
$$

Let $Q=\max \left\{\varepsilon+\alpha_{0}, \mu_{0}+\alpha_{0}, \mu_{0}+\lambda, \mu\right\}$, then $E(t) \leq 1+$ $Q \int_{0}^{t} E(s) \mathrm{d} s$. The Gronwall Inequality follows the estimation immediately: $E(t) \leq e^{Q T}$, for all $t \in[0, T]$. The proof of Lemma 6 is completed.

From Theorem 5, Lemma 6, and extension theorem, the existence and uniqueness of the system solution can be derived readily as below.

Theorem 7 (existence and uniqueness of global solution). For any $T>0$, the system (1)-(3) has a unique nonnegative solution $\left(P_{0}, P_{1}, \ldots, P_{4}, P_{5}\right) \in V_{0}^{5} \times V_{1}$.

3.3. Regularity of System Solution. In this section, we discuss the regularity or the $C^{1}$ continuity of the solution of system (1)-(3).

From Theorem 1 and the expressions in problem $(\mathrm{P})$ and noting the assumption (4), the following result is obvious. 
Theorem 8. For any $T>0$, if $\left(P_{0}, P_{1}, \ldots, P_{4}, P_{5}\right) \in V_{0}^{5} \times V_{1}$ is the nonnegative solution of system (1)-(3), then $P_{i} \in C[0, T]$, $i=0,1, \ldots, 4$ and $P_{5} \in C([0, T] \times[0, \infty))$.

Theorem 9. For any $T>0$, assume $\mu(x)$ is continuous on $[0, T]$. If $\left(P_{0}, P_{1}, \ldots, P_{4}, P_{5}\right) \in V_{0}^{5} \times V_{1}$ is the nonnegative solution of system (1)-(3), then $P_{i} \in C^{1}[0, T], i=0,1, \ldots, 4, P_{5} \in$ $C^{1}(D)$, where $D=\{(t, x) \mid 0<t \leq T, 0 \leq x<t\}$.

Proof. From Theorem 1 and the expressions in problem (P) combing the assumption (4), it is not difficult to know that for any $T>0$, if $\left(P_{0}, P_{1}, \ldots, P_{4}, P_{5}\right) \in V_{0}^{5} \times V_{1}$ is the nonnegative solution of system (1)-(3), then $P_{i}(t)$ is differentiable on $[0, T]$ and $P_{i}^{\prime}(t) \in C[0, T]$ by Theorem $8, i=0,1, \ldots, 4$. That is, $P_{i}(t) \in C^{1}[0, T], i=0,1, \ldots, 4$. And with the expression of $P_{5}(t, x)$ in problem $(\mathrm{P})$, we have

$$
\begin{gathered}
\left\{\begin{array}{lr}
\frac{\partial P_{5}(t, x)}{\partial t}=\left[\lambda P_{2}^{\prime}(t-x)+\mu_{0} P_{4}^{\prime}(t-x)\right] e^{-\int_{0}^{x} \mu(\tau) \mathrm{d} \tau}, \\
x<t \\
0, & x>t,
\end{array}\right. \\
\left\{\begin{array}{rr}
\frac{\partial P_{5}(t, x)}{\partial x}=\left[-\lambda P_{2}^{\prime}(t-x)-\mu_{0} P_{4}^{\prime}(t-x)\right] e^{-\int_{0}^{x} \mu(\tau) \mathrm{d} \tau} \\
-\mu(x)\left[\lambda P_{2}(t-x)+\mu_{0} P_{4}(t-x)\right] e^{-\int_{0}^{x} \mu(\tau) \mathrm{d} \tau}, \\
0, & x<t
\end{array}\right. \\
x>t .
\end{gathered}
$$

Then,

$$
\begin{gathered}
\left.\frac{\partial P_{5}(t, x)}{\partial t}\right|_{\substack{x<t \\
x \rightarrow t}}=\lambda \alpha_{0} e^{-\int_{0}^{x} \mu(\tau) \mathrm{d} \tau}, \\
\left.\frac{\partial P_{5}(t, x)}{\partial t}\right|_{\substack{x>t \\
x \rightarrow t}}=0, \\
\left.\frac{\partial P_{5}(t, x)}{\partial t}\right|_{\substack{x<t \\
x \rightarrow t}}=-\lambda \alpha_{0} e^{-\int_{0}^{x} \mu(\tau) \mathrm{d} \tau}, \\
\left.\frac{\partial P_{5}(t, x)}{\partial t}\right|_{\substack{x>t \\
x \rightarrow t}}=0 .
\end{gathered}
$$

Therefore, by the continuity of $P_{2}^{\prime}(t), P_{4}^{\prime}(t)$, and $\mu(x)$ on $[0, T]$, it can be yielded that $P_{5}(t, x) \in C^{1}(D)$, where $D=\{(t, x) \mid 0<$ $t \leq T, 0 \leq x<t\}$. The proof of Theorem 9 is completed.

\section{Stability of System Solution}

In this section, we will study the asymptotic stability and exponential stability of the solution of system (1)-(3). For convenience, we will first translate the system equations into an abstract Cauchy problem in a Banach space. Then, the asymptotic stability of the system solution is discussed by analyzing the spectral distributions of the system operator and that of its adjoint operator. Further, the exponential stability of the system solution is studied by analyzing the essential spectrum bound of the system operator.
4.1. System Transformation. In this section, we will translate the system equations into an abstract Cauchy problem in a suitable Banach space.

First, choose the state space $X$ to be

$$
\begin{gathered}
X=\left\{P=\left(P_{0}, P_{1}, \ldots, P_{4}, P_{5}(x)\right)^{\mathrm{T}} \mid P_{i} \in \mathbb{R}, i=0,1, \ldots, 4,\right. \\
\left.P_{5}(x) \in L^{1}\left(\mathbb{R}_{+}\right),\|P\|=\sum_{i=0}^{4}\left|P_{i}\right|+\left\|P_{5}\right\|_{L^{1}\left(\mathbb{R}_{+}\right)}<\infty\right\} .
\end{gathered}
$$

Here, $\mathbb{R}_{+}$denotes the set of nonnegative real numbers. Obviously, $X$ is a Banach space.

Next, define operator $A$ as follows:

$$
\begin{aligned}
& A P=\left(\begin{array}{c}
-\left(\varepsilon+\alpha_{0}\right) P_{0}+\mu_{0} P_{1}+\int_{0}^{\infty} \mu(x) P_{5}(x) \mathrm{d} x \\
-\left(\alpha_{0}+\mu_{0}\right) P_{1}+\varepsilon P_{0} \\
-\lambda P_{2}+\alpha_{0} P_{0}+\mu_{0} P_{3} \\
-\left(\lambda+\mu_{0}\right) P_{3}+\alpha_{0} P_{1} \\
-\mu_{0} P_{4}+\lambda P_{3} \\
-P_{5}^{\prime}(x)-\mu(x) P_{5}(x)
\end{array}\right) \\
& D(A)=\left\{P=\left(P_{0}, P_{1}, \ldots, P_{4}, P_{5}(x)\right)^{\mathrm{T}}\right. \\
& \in X \mid P_{5}^{\prime}(x) \in L^{1}\left(\mathbb{R}_{+}\right) ;
\end{aligned}
$$

$$
\left.P_{5}(0)=\lambda P_{2}+\mu_{0} P_{4}\right\} \text {. }
$$

Then, the system (1)-(3) can be rewritten as an abstract Cauchy problem in the Banach space $X$ :

$$
\begin{gathered}
\frac{\mathrm{d} P(t, \cdot)}{\mathrm{d} t}=A P(t, \cdot), \quad t \geq 0 \\
P(t, \cdot)=\left(P_{0}(t), \ldots, P_{4}(t), P_{5}(t, x)\right)^{\mathrm{T}} \\
P(0, \cdot) \triangleq P_{0}=(1,0, \ldots, 0)^{\mathrm{T}} .
\end{gathered}
$$

4.2. Properties of System Operator A. In this section, we will study some properties of the system operator $A$.

Lemma 10. The system operator $A$ is a densely closed dissipative operator.

Proof. Firstly, we prove that $A$ is a closed operator. Choose $P_{n}=\left(P_{n 0}, P_{n 1}, \ldots, P_{n 4}, P_{n 5}(x)\right)^{\mathrm{T}} \in D(A), P_{n} \rightarrow P=\left(P_{0}\right.$, $\left.P_{1}, \ldots, P_{4}, P_{5}(x)\right)^{\mathrm{T}}, A P_{n} \rightarrow Q=\left(Q_{0}, Q_{1}, \ldots, Q_{4}, Q_{5}(x)\right)^{\mathrm{T}}$, $n \rightarrow \infty$. By Proposition 1 ([29, II.2.10]), we know that the 
differential operator $\mathscr{D}$ is the infinitesimal generator of a left translation semigroup $\left\{T_{l}(t)\right\}_{t \geq 0}$ with domain

$D(\mathscr{D})$

$$
=\left\{f \in L^{1}\left(\mathbb{R}_{+}\right) \mid f\right.
$$

is absolutely continuous satisfying

$$
\left.f^{\prime} \in L^{1}\left(\mathbb{R}_{+}\right)\right\}
$$

Because $D(\mathscr{D})$ is closed and $P_{n 5} \in D(\mathscr{D})$, then $P_{5} \in D(\mathscr{D})$, that is, $P_{5}^{\prime}(x) \in L^{1}\left(\mathbb{R}_{+}\right)$, and $P_{5}(x)$ is absolutely continuous. Moreover, $P_{n 5}(0)=\lambda P_{2 n}+\mu_{0} P_{4 n} \rightarrow \lambda P_{2}+\mu_{0} P_{4}, n \rightarrow \infty$. Thus, $P \in D(A)$. Therefore, it is not difficult to get that $A P=$ $Q$ by noting the bounded measure of $\mu(x)$. This implies that $A$ is a closed operator.

Next, we prove that $D(A)$; the domain of $A$ is dense in $X$. For any $F=\left(F_{0}, F_{1}, \ldots, F_{4}, F_{5}(x)\right)^{\mathrm{T}} \in X$, let $P_{i}=F_{i}, i=$ $0,1, \ldots, 4$. Because $F_{5}(x) \in L^{1}\left(\mathbb{R}_{+}\right)$, then for any $\varepsilon>0$, there exist $\delta_{1}>0$ and $G>0$ such that

$$
\int_{0}^{\delta_{1}}\left|F_{5}(x)\right| \mathrm{d} x<\frac{\varepsilon}{6}, \quad \int_{G}^{\infty}\left|F_{5}(x)\right| \mathrm{d} x<\frac{\varepsilon}{3} .
$$

Set $\delta=\min \left\{\delta_{1}, 1 / 6\left(1+\left|\lambda P_{2}+\mu_{0} P_{4}\right|\right)\right\}$, and define

$$
P_{5}(x)= \begin{cases}\lambda P_{2}+\mu_{0} P_{4}, & 0 \leq x<\delta \\ g(x), & \delta \leq x \leq G \\ 0, & x>G .\end{cases}
$$

Here, $g(x)$ is continuously differentiable function on $[\delta, G]$ satisfying $g(\delta)=\lambda P_{5}+\mu P_{4}, g(G)=0$ and

$$
\int_{\delta}^{G}\left|P_{5}(x)-g(x)\right| \mathrm{d} x<\frac{\varepsilon}{3} .
$$

Choose $P=\left(P_{0}, P_{1}, \ldots, P_{4}, P_{5}(x)\right)^{\mathrm{T}}$, then $P \in D(A)$, and

$$
\begin{aligned}
\|P-F\|= & \int_{0}^{\delta}\left|P_{5}(x)-F_{5}(x)\right| \mathrm{d} x \\
& +\int_{\delta}^{G}\left|P_{5}(x)-F_{5}(x)\right| \mathrm{d} x \\
& +\int_{G}^{\infty}\left|P_{5}(x)-F_{5}(x)\right| \mathrm{d} x \\
< & \int_{0}^{\delta}\left|P_{5}(x)\right| \mathrm{d} x+\int_{0}^{\delta}\left|F_{5}(x)\right| \mathrm{d} x \\
& +\frac{\varepsilon}{3}+\frac{\varepsilon}{3}<\left(\lambda P_{2}+\mu_{0} P_{4}\right) \delta+\frac{\varepsilon}{6}+\frac{2 \varepsilon}{3} \\
< & \varepsilon .
\end{aligned}
$$

This implies that $D(A)$ is dense in $X$.

Thirdly, we prove that $A$ is a dissipative operator. In fact, for any $P=\left(P_{0}, P_{1}, \ldots, P_{4}, P_{5}(x)\right)^{\mathrm{T}} \in D(A)$, choose $Q=\left(Q_{0}, Q_{1}, \ldots, Q_{4}, Q_{5}(x)\right)^{\mathrm{T}}$, where $Q_{i}=\|P\| \operatorname{sgn}\left(P_{i}\right)$, $i=0,1, \ldots, 4, Q_{5}(x)=\|P\| \operatorname{sgn}\left(P_{5}(x)\right)$. Clearly, $Q \in X^{*}=$ $\mathbb{R}^{5} \times L^{\infty}\left(\mathbb{R}_{+}\right)$, the dual space of $X$, and $\langle P, Q\rangle=\|P\|^{2}=$ $\|Q\|^{2}$. Moreover, it is not difficult to know that $\langle A P, Q\rangle \leq 0$. This manifests that $A$ is a dissipative operator. The proof of Lemma 10 is completed.

Lemma 11. $\{\gamma \in \mathbb{C} \mid \operatorname{Re} \gamma>0$ or $\gamma=i a, a \in \mathbb{R} \backslash\{0\}\} \subset \rho(A)$, the resolvent set of the system operator $A$.

Proof. For any $G=\left(G_{0}, G_{1}, \ldots, G_{4}, G_{5}(x)\right)^{\mathrm{T}} \in X$, consider the operator equation $(\gamma I-A) P=G$. That is,

$$
\begin{gathered}
\left(\gamma+\varepsilon+\alpha_{0}\right) P_{0}=G_{0}+\mu_{0} P_{1}+\int_{0}^{\infty} \mu(x) P_{5}(x) \mathrm{d} x, \\
\left(\gamma+\alpha_{0}+\mu_{0}\right) P_{1}=G_{1}+\varepsilon P_{0}, \\
(\gamma+\lambda) P_{2}=G_{2}+\alpha_{0} P_{0}+\mu_{0} P_{3}, \\
\left(\gamma+\lambda+\mu_{0}\right) P_{3}=G_{3}+\alpha_{0} P_{1}, \\
\left(\gamma+\mu_{0}\right) P_{4}=G_{4}+\lambda P_{3}, \\
P_{5}^{\prime}(x)+(\gamma+\mu(x)) P_{5}(x)=G_{5}(x), \\
P_{5}(0)=\lambda P_{2}+\mu_{0} P_{4} .
\end{gathered}
$$

Solving (36) with the help of (37) yields

$$
\begin{aligned}
P_{5}(x)= & P_{5}(0) e^{-\int_{0}^{x}(\gamma+\mu(s)) \mathrm{d} s} \\
& +\int_{0}^{x} G_{5}(\tau) e^{-\int_{\tau}^{x}(\gamma+\mu(s)) \mathrm{d} s} \mathrm{~d} \tau \\
= & \left(\lambda P_{2}+\mu_{0} P_{4}\right) e^{-\int_{0}^{x}(\gamma+\mu(s)) \mathrm{d} s}+Y_{5}(x),
\end{aligned}
$$

where $Y_{5}(x)=\int_{0}^{x} G_{5}(\tau) e^{-\int_{\tau}^{x}[\gamma+\mu(s)] \mathrm{d} s} \mathrm{~d} \tau$. By [30], there exists a constant $N$, such that

$$
\int_{t}^{\infty} e^{-\int_{0}^{x} \mu(s) \mathrm{d} s} \mathrm{~d} x \leq N, \quad \forall t \geq 0
$$

Thus, $P_{5}(x) \in L^{1}\left(\mathbb{R}_{+}\right)$.

Substituting (38) into (31) derives

$$
\begin{aligned}
\left(\gamma+\varepsilon+\alpha_{0}\right) P_{0}= & G_{0}+\mu_{0} P_{1} \\
& +\int_{0}^{\infty} \mu(x) \\
& \times\left[P_{5}(0) e^{-\int_{0}^{x}(\gamma+\mu(s)) \mathrm{d} s}+Y_{5}(x)\right] \mathrm{d} x \\
= & \mu_{0} P_{1}+\left(\lambda P_{2}+\mu_{0} P_{4}\right) \\
& \times \int_{0}^{\infty} \mu(x) e^{-\int_{0}^{x}(\gamma+\mu(s)) \mathrm{d} s} \mathrm{~d} x+Y_{0},
\end{aligned}
$$

where $Y_{0}=G_{0}+\int_{0}^{\infty} \mu(x) Y_{5}(x) \mathrm{d} x$. 
Combing (40) and (32)-(35) follows the following matrix equation:

$$
\left(\begin{array}{ccccc}
\gamma+\varepsilon+\alpha_{0} & -\mu_{0} & -\lambda \int_{0}^{\infty} \mu(x) e_{0}^{\int_{0}^{x}(\gamma+\mu(s)) \mathrm{d} s} \mathrm{~d} x & 0 & -\mu_{0} \int_{0}^{\infty} \mu(x) e^{-\int_{0}^{x}(\gamma+\mu(s)) \mathrm{d} s} \mathrm{~d} x \\
-\varepsilon & \gamma+\alpha_{0}+\mu_{0} & 0 & 0 & 0 \\
-\alpha_{0} & 0 & \gamma+\lambda & -\mu_{0} & 0 \\
0 & -\alpha_{0} & 0 & \gamma+\lambda+\mu_{0} & 0 \\
0 & 0 & 0 & -\lambda & \gamma+\mu_{0}
\end{array}\right)\left(\begin{array}{c}
P_{0} \\
P_{1} \\
P_{2} \\
P_{3} \\
P_{4}
\end{array}\right)\left(\begin{array}{c}
Y_{0} \\
G_{1} \\
G_{2} \\
G_{3} \\
G_{4}
\end{array}\right)
$$

For $\operatorname{Re} \gamma>0$ or $\gamma=i a, a \in \mathbb{R} \backslash\{0\}$, it is not difficult to get the following estimation from the definition of modulus of complex number:

$$
\left|\int_{0}^{\infty} \mu(x) e^{-\int_{0}^{x}(\gamma+\mu(s)) \mathrm{d} s} \mathrm{~d} x\right|<1 .
$$

Thus, the coefficient matrix of the matrix equation (41) is a strictly diagonally dominant matrix for column. So, it is inverse, and the matric equation (41) has a unique solution $\left(P_{0}, P_{1}, P_{2}, P_{3}, P_{4}\right)^{\mathrm{T}}$. Combing (38), it can be seen that (31)(37) have a unique solution $P=\left(P_{0}, P_{1}, P_{2}, P_{3}, P_{4}, P_{5}(x)\right)^{\mathrm{T}} \in$
$D(A)$. This means that $\gamma I-A$ is surjective. Because $\gamma I-A$ is closed and $D(A)$ is dense in $X$, then $(\gamma I-A)^{-1}$ exists and is bounded by Inverse Operator Theorem, for any $\operatorname{Re} \gamma>0$ or $\gamma=i a, a \in \mathbb{R} \backslash\{0\}$. The proof of Lemma 11 is completed.

Lemma 12. 0 is an eigenvalue of the system operator $A$ with algebraic multiplicity one.

Proof. Consider the operator equation $(\gamma I-A) P=0$. Let $D(\gamma)$ be the determinant of coefficient of the matrix equation (41), then we have

$$
D(\gamma)=\left|\begin{array}{ccccc}
\gamma+\varepsilon+\alpha_{0} & -\mu_{0} & -\lambda(1-\gamma g(\gamma)) & 0 & -\mu_{0}(1-\gamma g(\gamma)) \\
-\varepsilon & \gamma+\alpha_{0}+\mu_{0} & 0 & 0 & 0 \\
-\alpha_{0} & 0 & \gamma+\lambda & -\mu_{0} & 0 \\
0 & -\alpha_{0} & 0 & \gamma+\lambda+\mu_{0} & 0 \\
0 & 0 & 0 & -\lambda & \gamma+\mu_{0}
\end{array}\right|=\gamma F(\gamma)
$$

Here, $g(\gamma)=\int_{0}^{\infty} e^{-\int_{0}^{x}(\gamma+\mu(s)) \mathrm{d} s}$ and

$$
\begin{gathered}
F(\gamma)=\alpha_{0} \lambda \mu_{0} g(\gamma)\left(\lambda+\mu_{0}\right)\left(\varepsilon+\alpha_{0}+\mu_{0}\right) \\
+\alpha_{0}\left(\varepsilon \mu_{0}^{2}+\mu^{3}+\mu_{0}^{2} \alpha_{0}\right) \\
+\lambda\left(\alpha_{0}+\mu_{0}\right)\left[\lambda\left(\mu_{0}+\varepsilon\right)\right. \\
\left.+\mu_{0}\left(\alpha_{0}+\varepsilon+\mu_{0}\right)\right] \\
+\alpha_{0} \lambda \gamma g(\gamma)\left(2 \varepsilon \mu_{0}+2 \alpha_{0} \mu_{0}\right. \\
\left.+3 \mu_{0}^{2}+2 \lambda \mu_{0}+\alpha_{0} \lambda\right) \\
+\gamma\left[\left(\lambda \mu_{0}+\lambda \alpha_{0}+\alpha_{0} \mu_{0}\right)\left(4 \mu_{0}+2 \varepsilon+\lambda\right)\right. \\
+\left(\lambda^{2}+\mu_{0}^{2}\right)\left(\mu_{0}+\varepsilon\right) \\
\left.+2 \mu_{0} \alpha_{0}^{2}+\varepsilon \lambda \mu_{0}+\lambda \alpha_{0}^{2}\right] \\
+\alpha_{0} \lambda \gamma^{2} g(\gamma)\left(3 \mu_{0}+\alpha_{0}+\lambda\right) \\
+\gamma^{2}\left(5 \lambda \mu_{0}+2 \varepsilon \lambda+\lambda^{2}+\alpha_{0}^{2}+3 \mu_{0}^{2}\right. \\
\left.+\varepsilon \alpha_{0}+5 \mu_{0} \alpha_{0}+3 \lambda \alpha_{0}+2 \varepsilon \mu_{0}\right)
\end{gathered}
$$

$$
\begin{aligned}
& +\gamma^{3}\left(2 \lambda+\varepsilon+2 \alpha_{0}+3 \mu_{0}+\alpha_{0} \lambda g(\gamma)\right) \\
& +\gamma^{4}
\end{aligned}
$$

Because

$$
\begin{aligned}
F(0)= & \alpha_{0} \lambda \mu_{0} g(0)\left(\lambda+\mu_{0}\right)\left(\varepsilon+\alpha_{0}+\mu_{0}\right) \\
& +\alpha_{0}\left(\varepsilon \mu_{0}^{2}+\mu^{3}+\mu_{0}^{2} \alpha_{0}\right)+\lambda\left(\alpha_{0}+\mu_{0}\right) \\
& \times\left[\lambda\left(\mu_{0}+\varepsilon\right)+\mu_{0}\left(\alpha_{0}+\varepsilon+\mu_{0}\right)\right]>0 .
\end{aligned}
$$

This means $\gamma=0$ is an eigenvalue of the system operator $A$ with algebraic multiplicity one. The proof of Lemma 12 is completed.

4.3. Properties of Adjoint Operator $A^{*}$. In this section, we will study some properties of $A^{*}$, the adjoint operator of system operator $A$.

The dual space of $X$ is

$$
X^{*}=\mathbb{R}^{5} \times L^{\infty}\left(\mathbb{R}_{+}\right)
$$

with norm $\|Q\|=\sup \left\{\left|Q_{i}\right|,\left\|Q_{5}\right\|_{L^{\infty}\left(\mathbb{R}_{+}\right)}, i=0,1, \ldots, 4\right\}$ for $Q=$ $\left(Q_{0}, Q_{1}, \ldots, Q_{4}, Q_{5}(x)\right)^{\mathrm{T}} \in X^{*}$. 
Lemma 13. $A^{*}$, the adjoint operator of the system operator $A$ is as follows:

$$
A^{*} Q=\left(\begin{array}{c}
-\left(\varepsilon+\alpha_{0}\right) Q_{0}+\varepsilon Q_{1}+\alpha_{0} Q_{2} \\
-\left(\alpha_{0}+\mu_{0}\right) Q_{1}+\mu_{0} Q_{0}+\alpha_{0} Q_{3} \\
-\lambda Q_{2}+\lambda Q_{5}(0) \\
-\left(\lambda+\mu_{0}\right) Q_{3}+\mu_{0} Q_{2}+\lambda Q_{4} \\
-\mu_{0} Q_{4}+\mu_{0} Q_{5}(0) \\
Q_{5}^{\prime}(x)+\mu(x)\left[Q_{0}-Q_{5}(x)\right]
\end{array}\right) \triangleq(C+D) Q
$$

with domain

$$
\begin{aligned}
D\left(A^{*}\right) & \\
=\{Q & =\left(Q_{0}, Q_{1}, \ldots, Q_{4}, Q_{5}(x)\right)^{\mathrm{T}} \\
& \in X^{*} \mid Q_{5}^{\prime}(x) \in L^{\infty}\left(\mathbb{R}_{+}\right), Q_{5}(x)
\end{aligned}
$$

is absolutely continuous satisfying

$$
\left.Q_{5}(\infty)<\infty\right\}
$$

Here,

$$
\begin{gathered}
C Q=\operatorname{diag}\left(-\left(\varepsilon+\alpha_{0}\right),-\left(\alpha_{0}+\mu_{0}\right),\right. \\
\left.-\lambda,-\left(\lambda+\mu_{0}\right),-\mu_{0}, \frac{\mathrm{d}}{\mathrm{d} x}-\mu(x)\right) Q, \\
D Q=\left(\begin{array}{cccccc}
0 & \varepsilon & \alpha_{0} & 0 & 0 & 0 \\
\mu_{0} & 0 & 0 & \alpha_{0} & 0 & 0 \\
0 & 0 & 0 & 0 & 0 & \lambda \theta(\cdot) \\
0 & 0 & \mu_{0} & 0 & \lambda & 0 \\
0 & 0 & 0 & 0 & 0 & \mu_{0} \theta(\cdot) \\
\mu(x) & 0 & 0 & 0 & 0 & 0
\end{array}\right) Q
\end{gathered}
$$

and $\theta(\cdot): L^{\infty}\left(\mathbb{R}_{+}\right) \rightarrow \mathbb{C}$, satisfying $\theta(f)=f(0)$.

Proof. For any $P \in D(A)$ and $Q \in X^{*}, A^{*}$ and its domain $D\left(A^{*}\right)$ can be readily derived by the equality $\langle A P, Q\rangle=$ $\left\langle P, A^{*} Q\right\rangle$. The proof of Lemma 13 is completed.

Lemma 14. $S=\left\{\gamma \in \mathbb{C} \mid \sup \left\{\left(\varepsilon+\alpha_{0}\right) /\left|\gamma+\varepsilon+\alpha_{0}\right|,\left(\mu_{0}+\right.\right.\right.$ $\left.\alpha_{0}\right) /\left|\gamma+\mu_{0}+\alpha_{0}\right|, \lambda /|\gamma+\lambda|,\left(\lambda+\mu_{0}\right) /\left|\gamma+\lambda+\mu_{0}\right|, \mu_{0} /\left|\gamma+\mu_{0}\right|$, $M /(\operatorname{Re} \gamma+M)\}<1\} \subset \rho\left(A^{*}\right)$, the resolvent set of $A^{*}$, where $M=\sup _{x \geq 0} \mu(x)$.

Proof. For any $W=\left(W_{0}, W_{1}, \ldots, W_{4}, W_{5}(x)\right)^{\mathrm{T}} \in X^{*}$, consider the equation $(\gamma I-C) Q=D W$. That is,

$$
\begin{gathered}
\left(\gamma+\varepsilon+\alpha_{0}\right) Q_{0}=\varepsilon W_{1}+\alpha_{0} W_{2}, \\
\left(\gamma+\alpha_{0}+\mu_{0}\right) Q_{1}=\mu_{0} W_{0}+\alpha_{0} W_{3},
\end{gathered}
$$

$$
\begin{gathered}
(\gamma+\lambda) Q_{2}=\lambda W_{5}(0), \\
\left(\gamma+\lambda+\mu_{0}\right) Q_{3}=\mu_{0} W_{2}+\lambda W_{4}, \\
\left(\gamma+\mu_{0}\right) Q_{4}=\mu_{0} W_{5}(0), \\
\frac{\mathrm{d}_{5}(x)}{\mathrm{d} x}=(\gamma+\mu(x)) Q_{5}(x)-\mu(x) W_{0} .
\end{gathered}
$$

Solving (50)-(54) yields

$$
Q_{0}=\frac{\varepsilon W_{1}+\alpha_{0} W_{2}}{\gamma+\varepsilon+\alpha_{0}}, \quad Q_{1}=\frac{\mu_{0} W_{0}+\alpha_{0} W_{3}}{\gamma+\alpha_{0}+\mu_{0}},
$$

$$
\begin{gathered}
Q_{2}=\frac{\lambda W_{5}(0)}{\gamma+\lambda}, \\
Q_{3}=\frac{\mu_{0} W_{2}+\lambda W_{4}}{\gamma+\lambda+\mu_{0}}, \quad Q_{4}=\frac{\mu_{0} W_{5}(0)}{\gamma+\mu_{0}} .
\end{gathered}
$$

Solving (55) derives

$$
Q_{5}(x)=e^{\int_{0}^{x}(\gamma+\mu(s)) \mathrm{d} s}\left[Q_{5}(0)-\int_{0}^{x} W_{0} \mu(\tau) e^{-\int_{0}^{\tau}(\gamma+\mu(s)) \mathrm{d} s} \mathrm{~d} \tau\right]
$$

multiplying $e^{-\int_{0}^{x}(\gamma+\mu(s)) \mathrm{d} s}$ to the two sides of (57) and letting $x \rightarrow \infty$ by noting $Q_{5}(\infty)<\infty$ follows

$$
Q_{5}(0)=\int_{0}^{\infty} W_{0} \mu(\tau) e^{-\int_{0}^{\tau}(\gamma+\mu(s)) \mathrm{d} s} \mathrm{~d} \tau .
$$

Substituting (58) into (57) yields

$$
Q_{5}(x)=e^{\int_{0}^{x}(\gamma+\mu(s)) \mathrm{d} s} \int_{x}^{\infty} W_{0} \mu(\tau) e^{-\int_{0}^{\tau}(\gamma+\mu(s)) \mathrm{d} s} \mathrm{~d} \tau .
$$

Thus, we can get the following estimation:

$$
\begin{aligned}
& \left\|Q_{5}\right\|_{L^{\infty}[0, \infty)} \\
& =\sup _{x \in[0, \infty)}\left|Q_{5}(x)\right| \\
& =\sup _{x \in[0, \infty)} \mid e^{\int_{0}^{x}(\gamma+\mu(s)) \mathrm{d} s} \\
& \quad \times \int_{x}^{\infty} W_{0} \mu(\tau) e^{-\int_{0}^{\tau}(\gamma+\mu(s)) \mathrm{d} s} \mathrm{~d} \tau \mid \\
& \leq\|W\| \sup _{x \in[0, \infty)} e^{\int_{0}^{x}(\operatorname{Re} \gamma+\mu(s)) \mathrm{d} s} \\
& \quad \times \int_{x}^{\infty}-e^{-\operatorname{Re} \gamma \tau} \mathrm{d} e^{-\int_{0}^{\tau} \mu(s) \mathrm{d} s}
\end{aligned}
$$




$$
\begin{aligned}
& =\|W\| \sup _{x \in[0, \infty)} e^{\int_{0}^{x}(\operatorname{Re} \gamma+\mu(s)) \mathrm{d} s} \\
& \times\left[e^{-\int_{0}^{x}(\operatorname{Re} \gamma+\mu(s)) \mathrm{d} s}\right. \\
& \left.-\operatorname{Re} \gamma \int_{x}^{\infty} e^{-\int_{0}^{\tau}(\operatorname{Re} \gamma+\mu(s)) \mathrm{d} s} \mathrm{~d} \tau\right] \\
& =\|W\| \sup _{x \in[0, \infty)}\left[1-\operatorname{Re} \gamma e^{\operatorname{Re} \gamma x}\right. \\
& \left.\times \int_{x}^{\infty} e^{-\int_{x}^{\tau}(\operatorname{Re} \gamma+\mu(s)) \mathrm{d} s} \mathrm{~d} \tau\right] \\
& \leq\|W\| \sup _{x \in[0, \infty)}\left[1-\operatorname{Re} \gamma e^{\operatorname{Re} \gamma x}\right. \\
& \left.\times \int_{x}^{\infty} e^{-\int_{x}^{\tau}(\operatorname{Re} \gamma+M) \mathrm{d} s} \mathrm{~d} \tau\right] \\
& =\|W\| \sup _{x \in[0, \infty)}\left[1-\operatorname{Re} \gamma e^{(\operatorname{Re} \gamma+M) x}\right. \\
& \left.\times \int_{x}^{\infty} e^{-(\operatorname{Re} \gamma+M) \tau} \mathrm{d} \tau\right] \\
& =\|W\| \sup _{x \in[0, \infty)}\left[1-\operatorname{Re} \gamma e^{(\operatorname{Re} \gamma+M) x}\right. \\
& \left.\times \frac{e^{-(\operatorname{Re} \gamma+M) x}}{\operatorname{Re} \gamma+M}\right] \\
& =\frac{M}{\operatorname{Re} \gamma+M}\|W\|,
\end{aligned}
$$

where $M=\sup _{x \geq 0} \mu(x)$.

Equation (56) derive the following estimations:

$$
\begin{array}{cc}
\left|Q_{0}\right| \leq \frac{\varepsilon+\alpha_{0}}{\left|\gamma+\varepsilon+\alpha_{0}\right|}\|W\|, & \left|Q_{1}\right| \leq \frac{\mu_{0}+\alpha_{0}}{\left|\gamma+\alpha_{0}+\mu_{0}\right|}\|W\|, \\
\left|Q_{2}\right| \leq \frac{\lambda}{|\gamma+\lambda|}\|W\|, & \left|Q_{3}\right| \leq \frac{\lambda+\mu_{0}}{\left|\gamma+\lambda+\mu_{0}\right|}\|W\|, \\
\left|Q_{4}\right| \leq \frac{\mu_{0}}{\left|\gamma+\mu_{0}\right|}\|W\|, & \left|Q_{5}\right| \leq \frac{M}{\operatorname{Re} \gamma+M}\|W\| .
\end{array}
$$

Then, for $\gamma \in S$, we have

$$
\begin{array}{r}
\|Q\|=\sum_{i=0}^{4}\left|Q_{i}\right|+\left\|Q_{5}\right\| \\
\leq \sup \left\{\frac{\varepsilon+\alpha_{0}}{\left|\gamma+\varepsilon+\alpha_{0}\right|}, \frac{\mu_{0}+\alpha_{0}}{\left|\gamma+\alpha_{0}+\mu_{0}\right|},\right. \\
\frac{\lambda}{|\gamma+\lambda|}, \frac{\lambda+\mu_{0}}{\left|\gamma+\lambda+\mu_{0}\right|},
\end{array}
$$

$$
\left.\frac{\mu_{0}}{\left|\gamma+\mu_{0}\right|}, \frac{M}{\operatorname{Re} \gamma+M}\right\}\|W\|
$$$$
<\|W\| \text {. }
$$

This implies that $\left\|(\gamma I-C)^{-1} D\right\|<1$. Thus, $\left[I-(\gamma I-C)^{-1} D\right]$ is invertible. Therefore, $\left(\gamma I-A^{*}\right)$ is invertible and

$$
\begin{aligned}
\left(\gamma I-A^{*}\right)^{-1} & =[\gamma I-(C+D)]^{-1} \\
& =\left[I-(\gamma I-C)^{-1} D\right]^{-1}(\gamma I-C)^{-1}, \\
& \gamma \in S .
\end{aligned}
$$

The proof of Lemma 14 is completed.

Lemma 15. 0 is an eigenvalue of operator $A^{*}$ with algebraic multiplicity one.

Proof. We prove Lemma 15 in two steps. Firstly, we prove that 0 is an eigenvalue of operator $A^{*}$. For any $Q \in D\left(A^{*}\right)$, consider the operator equation $A^{*} Q=0$, that is,

$$
\begin{gathered}
\left(\varepsilon+\alpha_{0}\right) Q_{0}=\varepsilon Q_{1}+\alpha_{0} Q_{2}, \\
\left(\alpha_{0}+\mu_{0}\right) Q_{1}=\mu_{0} Q_{0}+\alpha_{0} Q_{3}, \\
\lambda Q_{2}=\lambda Q_{5}(0), \\
\left(\lambda+\mu_{0}\right) Q_{3}=\mu_{0} Q_{2}+\lambda Q_{4}, \\
\mu_{0} Q_{4}=\mu_{0} Q_{5}(0), \\
Q_{5}^{\prime}(x)=\mu(x) Q_{5}(x)-Q_{0} \mu(x) .
\end{gathered}
$$

Solving (69) yields

$$
Q_{5}(x)=e^{\int_{0}^{x} \mu(s) \mathrm{d} s}\left[Q_{5}(0)-Q_{0} \int_{0}^{x} \mu(\tau) e^{-\int_{0}^{\tau} \mu(s) \mathrm{d} s} \mathrm{~d} \tau\right]
$$

multiplying $e^{-\int_{0}^{x} \mu(s) \mathrm{d} s}$ to the two sides of (70) and letting $x \rightarrow$ $\infty$ by noting $Q_{5}(\infty)<\infty$ derive

$$
Q_{5}(0)=Q_{0} \int_{0}^{\infty} \mu(\tau) e^{-\int_{0}^{\tau} \mu(s) \mathrm{d} s} \mathrm{~d} \tau=Q_{0} .
$$

Combing (64)-(68) with (71) follows

$$
Q_{0}=Q_{1}=Q_{2}=Q_{3}=Q_{4}=Q_{5}(0) .
$$

Substituting (71) into (70) yields

$$
Q_{5}(x)=Q_{0} e^{\int_{0}^{x} \mu(s) \mathrm{d} s}\left[1+\int_{0}^{x} \mathrm{~d} e^{-\int_{0}^{\tau} \mu(s) \mathrm{d} s}\right]=Q_{0} .
$$

This implies that $\eta Q^{*}(\eta \neq 0)$ is the eigenfunction corresponding to eigenvalue 0 of operator $A^{*}$, where $Q^{*}=$ $(1,1, \ldots, 1)^{\mathrm{T}}$.

Next, we prove that the algebraic multiplicity of 0 in $X^{*}$ is one. From the above step, we can see that the geometric 
multiplicity of 0 in $X^{*}$ is one. Then, we only need to verify the algebraic index of 0 in $X^{*}$ which is also one according to [31]. We use the reduction to absurdity. Suppose that the algebraic index of 0 in $X^{*}$ is 2 without loss of generality, then there exists $Y \in X^{*}$, such that $A^{*} Y=Q^{*}$. It is obvious that

$$
0=\langle A P, Y\rangle=\left\langle P, A^{*} Y\right\rangle=\left\langle P, Q^{*}\right\rangle,
$$

where $P$ is the positive eigenfunction corresponding to eigenvalue 0 of $A$. However,

$$
\left\langle P, Q^{*}\right\rangle=\sum_{i=0}^{4} P_{i}+\int_{0}^{\infty} P_{5}(x) \mathrm{d} x>0
$$

which contradicts (74). Thus, the algebraic index of 0 in $X^{*}$ is one. Therefore, the algebraic multiplicity of 0 in $X^{*}$ is one. The proof of Lemma 15 is completed.

4.4. Asymptotic Stability of System Solution. In this section, we will present the asymptotic stability of the system solution by using $C_{0}$ semigroup theory.

Recalling Phillips Theorem (see [32]) together with Lemmas 10,11 , and 3 , we can obtain the following results.

Theorem 16. The system operator $A$ generates a positive $C_{0}$ semigroup of contraction $T(t)$.

Theorem 17. The system (25) has a unique nonnegative timedependent solution $P(t, \cdot)$ which satisfies

$$
\|P(t, \cdot)\|=1, \quad \forall t \in[0, \infty) .
$$

Proof. From Theorem 16 and [32], it can be derived that the system (25) has a unique nonnegative solution $P(t, \cdot)$ which can be expressed as

$$
P(t, \cdot)=T(t) P_{0}, \quad \forall t \in[0, \infty) .
$$

Because $P(t, \cdot)$ satisfies (1)-(3), it is not difficult to know that

$$
\frac{\mathrm{d}\|P(t, \cdot)\|}{\mathrm{d} t}=0 \text {. }
$$

Therefore,

$$
\|P(t, \cdot)\|=\left\|T(t) P_{0}\right\|=\left\|P_{0}\right\|=1, \quad \forall t \in[0, \infty) .
$$

This just reflects the physical meaning of $P(t, \cdot)$. The proof of Theorem 17 is completed.

Remark 18. Because the initial value $P_{0}$ of the system (25) belongs to the domain $D(A)$ of the system operator $A$, then the nonnegative time-dependent solution of the system expressed in (77) is the strong solution of the system (25).

Noting that the $C_{0}$ semigroup $T(t)$ generated by $A$ is contractive according to Theorem 16 , it is uniformly bounded certainly. Thus, recalling [33] combining Lemmas 11, 12, 14, and 15 , we can know that the time-dependent solution of the system strongly converges to its steady-state solution. That is the following result.
Theorem 19. Let $\widehat{P}$ be the eigenfunction corresponding to eigenvalue 0 of the system operator A satisfying $\|\widehat{P}\|=1$, and let $Q^{*}$ be defined in Lemma 15, then the time-dependent solution $P(t, \cdot)$ of the system (25) converges to the nonnegative steadystate solution $\widehat{P}$. That is,

$$
\lim _{t \rightarrow \infty} P(t, \cdot)=\left\langle P_{0}, Q^{*}\right\rangle \widehat{P}=\widehat{P},
$$

where $P_{0}$ is the initial value of the system.

4.5. Exponential Stability of System Solution. In Section 4.4, we have obtained the asymptotic stability of the system. In other words, the dynamic solution of the system asymptotically converges to its steady-state solution. However, there are still two problems: first, the convergence rate is unknown; second, the convergence is subject to some factors such as failure rate and repair rate. Both can be well settled if the system is exponentially stable. For this purpose, in this section, we will discuss the exponential stability of the system.

For simplicity, we will divide the system operator $A$ into two operators. The one is a compact operator, and the other generates a quasicompact semigroup. Then, by the perturbation of compact operator, it is derived readily that the system operator also generates a quasicompact $C_{0}$ semigroup. Therefore, the system solution is exponentially stable.

For convenience, we will introduce three operators first:

$$
\begin{gathered}
B P=\left(\int_{0}^{\infty} \mu(x) P_{5}(x) \mathrm{d} x+\mu_{0} P_{1}, \varepsilon P_{0}, \alpha_{0} P_{0}\right. \\
\left.+\mu_{0} P_{3}, \alpha_{0} P_{1}, \lambda P_{3}, 0\right)^{\mathrm{T}} \text { with } D(B)=X, \\
\bar{A}=A-B \text { with } D(\bar{A})=D \\
=\left\{\begin{array}{c}
P=\left(P_{0}, P_{1}, \ldots, P_{4}, P_{5}(x)\right)^{\mathrm{T}} \in X \mid P_{5}^{\prime}(x) \in L^{1}\left(\mathbb{R}_{+}\right), \\
P_{5}(x) \text { is an absolutely continuous function }
\end{array}\right\} \\
A_{0}=\bar{A} \quad \text { with } D\left(A_{0}\right)=\left\{P \in D \mid P_{5}(0)=0\right\} .
\end{gathered}
$$

It is easy to know that $\bar{A}$ and $A_{0}$ are both closed operators with dense domains in $X$. And with the perturbation of $C_{0}$ semigroup, it is clear that $\bar{A}$ also generates a $C_{0}$ semigroup $S(t)$.

Lemma 20. Assume that the mean of the repair rate exists and greater than zero, that is,

$$
0<\widehat{\mu}=\lim _{x \rightarrow \infty} \frac{1}{x} \int_{0}^{x} \mu(s) \mathrm{d} s .
$$

Then, $A_{0}$ generates a quasicompact semigroup $T_{0}(t)$.

Proof. Firstly, we will prove that $A_{0}$ generates a $C_{0}$ semigroup $T_{0}(t)$. Consider the following abstract Cauchy problem:

$$
\begin{gathered}
\frac{\mathrm{d} P(t, \cdot)}{\mathrm{d} t}=A_{0} P(t, \cdot), \quad t \geq 0, \\
P(0, \cdot)=\Phi,
\end{gathered}
$$


where $\Phi=\left(\varphi_{0}, \varphi_{1}, \ldots, \varphi_{4}, \varphi_{5}(x)\right)^{\mathrm{T}} \in X$. That is,

$$
\begin{gathered}
\left(\frac{\mathrm{d}}{\mathrm{d} t}+\varepsilon+\alpha_{0}\right) P_{0}(t)=0, \\
\left(\frac{\mathrm{d}}{\mathrm{d} t}+\alpha_{0}+\mu_{0}\right) P_{1}(t)=0, \\
\left(\frac{\mathrm{d}}{\mathrm{d} t}+\lambda\right) P_{2}(t)=0, \\
\left(\frac{\mathrm{d}}{\mathrm{d} t}+\lambda+\mu_{0}\right) P_{3}(t)=0, \\
\left(\frac{\mathrm{d}}{\mathrm{d} t}+\mu_{0}\right) P_{4}(t)=0, \\
{\left[\frac{\partial}{\partial t}+\frac{\partial}{\partial x}+\mu(x)\right] P_{5}(t, x)=0,} \\
P_{5}(t, 0)=0, \\
P_{i}(0)=\varphi_{i}, \quad i=0,1, \ldots, 4, \\
P_{5}(0, x)=\varphi_{5}(x) .
\end{gathered}
$$

Solving (84)-(88) with the help of (91) yields

$$
\begin{gathered}
P_{0}(t)=\varphi_{0} e^{-\left(\varepsilon+\alpha_{0}\right) t}, \quad P_{1}(t)=\varphi_{1} e^{-\left(\alpha_{0}+\mu_{0}\right) t}, \\
P_{2}(t)=\varphi_{2} e^{-\lambda t}, \\
P_{3}(t)=\varphi_{3} e^{-\left(\lambda+\mu_{0}\right) t}, \quad P_{4}(t)=\varphi_{4} e^{-\mu_{0} t} .
\end{gathered}
$$

Solving (89) with the help of (90) and (92) by the method of characteristics yields

$$
P_{5}(t, x)=\left\{\begin{array}{rr}
P_{5}(t-x, 0) e^{-\int_{0}^{x} \mu(\tau) \mathrm{d} \tau}=0, & t>x \\
P_{5}(0, x-t) e^{-\int_{0}^{t} \mu(x-t+\tau) \mathrm{d} \tau} & \\
=\varphi_{5}(x-t) e^{-\int_{x-t}^{x} \mu(\tau) \mathrm{d} \tau}, & t \leq x .
\end{array}\right.
$$

Therefore, it is easy to prove that $A_{0}$ generates a $C_{0}$ semigroup $T_{0}(t)$ satisfying

$$
\left(T_{0}(t) \Phi\right)(x)= \begin{cases}\left(\Psi_{1}, 0\right)^{\mathrm{T}}, & x<t \\ \left(\Psi_{1}, \varphi_{5}(x-t) e^{-\int_{x-t}^{x} \mu(\tau) \mathrm{d} \tau}\right)^{\mathrm{T}}, & x \geq t .\end{cases}
$$

Here,

$$
\Psi_{1}=\left(\varphi_{0} e^{-\left(\varepsilon+\alpha_{0}\right) t}, \varphi_{1} e^{-\left(\alpha_{0}+\mu_{0}\right) t}, \varphi_{2} e^{-\lambda t}, \varphi_{3} e^{-\left(\lambda+\mu_{0}\right) t}, \varphi_{4} e^{-\mu_{0} t}\right) .
$$

Next, we will prove that $T_{0}(t)$ is quasicompact. We only need to prove that the essential growth bound $W_{\text {ess }}\left(A_{0}\right)$ is less than zero.

The assumption condition (82) implies that for any $\varepsilon>0$, there exists $t_{0}>0$ such that

$$
\frac{1}{t} \int_{x-t}^{x} \mu(s) \mathrm{d} s>\widehat{\mu}-\varepsilon, \quad x \geq t \geq t_{0} .
$$

With the help of (97), it is not difficult to deduce that

$$
\begin{aligned}
\left\|T_{0}(t) \Phi\right\|= & \left|\varphi_{0}\right| e^{-\left(\varepsilon+\alpha_{0}\right) t}+\left|\varphi_{1}\right| e^{-\left(\alpha_{0}+\mu_{0}\right) t} \\
& +\left|\varphi_{2}\right| e^{-\lambda t}+\left|\varphi_{3}\right| e^{-\left(\lambda+\mu_{0}\right) t} \\
& +\left|\varphi_{4}\right| e^{-\mu_{0} t} \\
& +\int_{t}^{\infty}\left|\varphi_{5}(x-t)\right| e^{-\int_{x-t}^{x} \mu(\tau) \mathrm{d} \tau} \mathrm{d} x \\
< & \left|\varphi_{0}\right| e^{-\left(\varepsilon+\alpha_{0}\right) t}+\left|\varphi_{1}\right| e^{-\left(\alpha_{0}+\mu_{0}\right) t} \\
& +\left|\varphi_{2}\right| e^{-\lambda t}+\left|\varphi_{3}\right| e^{-\left(\lambda+\mu_{0}\right) t} \\
& +\left|\varphi_{4}\right| e^{-\mu_{0} t} \\
& +\int_{t}^{\infty}\left|\varphi_{5}(x-t)\right| e^{-(\widehat{\mu}-\varepsilon) t} \mathrm{~d} x \\
= & \left|\varphi_{0}\right| e^{-\left(\varepsilon+\alpha_{0}\right) t}+\left|\varphi_{1}\right| e^{-\left(\alpha_{0}+\mu_{0}\right) t} \\
& +\left|\varphi_{2}\right| e^{-\lambda t}+\left|\varphi_{3}\right| e^{-\left(\lambda+\mu_{0}\right) t} \\
& +\left|\varphi_{4}\right| e^{-\mu_{0} t} \\
& +e^{-(\hat{\mu}-\varepsilon) t} \int_{0}^{\infty}\left|\varphi_{5}(x)\right| \mathrm{d} x \\
\leq & e^{-\min \left\{\varepsilon+\alpha_{0}, \alpha_{0}+\mu_{0}, \lambda, \lambda+\mu_{0}, \mu_{0}, \widehat{\mu}-\varepsilon\right\} t}\|\Phi\| .
\end{aligned}
$$

This manifests that

$$
\left\|T_{0}(t)\right\| \leq e^{-\min \left\{\varepsilon+\alpha_{0}, \lambda, \mu_{0}, \widehat{\mu}-\varepsilon\right\} t} .
$$

Then,

$$
\begin{aligned}
W_{\text {ess }}\left(A_{0}\right) & \leq W\left(A_{0}\right)=\lim _{t \rightarrow \infty} \frac{\ln \left\|T_{0}(t)\right\|}{t} \\
& \leq-\min \left\{\varepsilon+\alpha_{0}, \lambda, \mu_{0}, \widehat{\mu}-\varepsilon\right\}<0 .
\end{aligned}
$$

Therefore, $A_{0}$ generates a quasicompact $C_{0}$ semigroup $T_{0}(t)$. The proof of Lemma 20 is completed.

$$
\begin{aligned}
& \text { For } \gamma>0, P \in X \text {, let } \\
& \Phi_{\gamma}(P)(x)=\left[\operatorname{diag}\left(0,0,0,0,0, \lambda P_{2}+\mu_{0} P_{4}\right)\right] \cdot E_{\gamma}(x),
\end{aligned}
$$

where $E_{\gamma}(x)=\left(0,0,0,0,0, e^{-\int_{0}^{x}[\gamma+\mu(s)] \mathrm{d} s}\right)^{\mathrm{T}} \in \operatorname{Ker}(\gamma I-\bar{A})$ and $\Phi_{\gamma}$ is a compact operator. Then, it is not difficult to obtain the following result.

Lemma 21. $I+\Phi_{\gamma}$ is a bijection from $D\left(A_{0}\right)$ to $D(A)$ and

$$
[\gamma I-(A-B)]\left(I+\Phi_{\gamma}\right)=\gamma I-A_{0} .
$$

Lemma 22. $S(t)-T_{0}(t)$ is a compact operator, for any $t \geq 0$. Here $S(t)$ is the $C_{0}$ semigroup generated by $\bar{A}$.

Proof. From Lemma 21, we can see that $R(\gamma, A-B) \geq$ $R\left(\gamma, A_{0}\right)$, for any $\gamma>0$. Therefore $S(t) \geq T_{0}(t)$, for any $t \geq 0$. 
For $P \in D\left(A_{0}\right)$, set

$$
\Psi(s) P=S(t-s)\left(I+\Phi_{\gamma}\right) T_{0}(s) P
$$

where $0 \leq s \leq t, \gamma>0$. Recalling the properties of $C_{0^{-}}$ semigroup and Lemma 21, we can obtain

$$
\begin{aligned}
\Psi^{\prime}(s) P= & -S(t-s)(A-B)\left(I+\Phi_{\gamma}\right) T_{0}(s) P \\
& +S(t-s)\left(I+\Phi_{\gamma}\right) A_{0} T_{0}(s) P \\
= & S(t-s)[\gamma I-(A-B)]\left(I+\Phi_{\gamma}\right) T_{0}(s) P \\
& +S(t-s)\left(I+\Phi_{\gamma}\right)\left[-\gamma I+A_{0}\right] T_{0}(s) P \\
= & S(t-s)\left[\gamma I-A_{0}\right] T_{0}(s) P \\
& +S(t-s)\left(I+\Phi_{\gamma}\right)\left(-\gamma I+A_{0}\right) T_{0}(s) P \\
= & S(t-s) \Phi_{\gamma}\left(-\gamma I+A_{0}\right) T_{0}(s) P .
\end{aligned}
$$

Since $[\Psi(t)-\Psi(0)] P=\int_{0}^{t} \Psi^{\prime}(s) P \mathrm{~d} s$, then

$$
[\Psi(t)-\Psi(0)] P=\int_{0}^{t} S(t-s) \Phi_{\gamma}\left(-\gamma I+A_{0}\right) T_{0}(s) P \mathrm{~d} s .
$$

That is,

$$
\begin{aligned}
S(t) P-T_{0}(t) P=- & \int_{0}^{t} S(t-s) \Phi_{\gamma}\left(-\gamma I+A_{0}\right) \\
& \times T_{0}(s) P \mathrm{~d} s+\Phi_{\gamma} T_{0}(t) P-S(t) \Phi_{\gamma} P .
\end{aligned}
$$

Therefore, $S(t)-T_{0}(t)(t \geq 0)$ is compact because the righthand side of the above equation is the sum of three compact operators for the compactness of $\Phi_{\gamma}$. The proof of Lemma 22 is completed.

From the above preparations, we can present the main results of this section.

Theorem 23. $C_{0}$ semigroup $T(t)$ generated by the system operator $A$ is quasicompact.

Proof. According to Proposition 9.20 (see [34]) combing Lemmas 22 and 20, we can deduce that

$$
W_{\text {ess }}(\bar{A}) \leq W\left(A_{0}\right)<0 .
$$

This shows that $S(t)$, the $C_{0}$ semigroup generated by $\bar{A}$ is quasicompact. Because $B$ is a compact operator, then according to [35], it is evident that

$$
W_{\text {ess }}(A)=W_{\text {ess }}(A-B)<0 .
$$

This implies that $T(t)$ is quasicompact. The proof of Theorem 23 is completed.
Theorem 24. The time-dependent solution of the system (1)(3) strongly converges to its steady-state solution. That is,

$$
\lim _{t \rightarrow \infty} P(t, \cdot)=\widehat{P} \text {. }
$$

Moreover, there exist $C>0$ and $\varepsilon>0$ such that

$$
\|P(t, \cdot)-\widehat{P}\| \leq C e^{-\varepsilon t} \text {. }
$$

Here, $\widehat{P}$ is defined in Theorem 19.

Proof. Recalling Theorem 2.10 (see [35]) combing Theorem 23, we can derive that $C_{0}$ semigroup $T(t)$ generated by the system operator $A$ can be decomposed as $T(t)=\bar{P}_{0}+R(t)$, where $\bar{P}_{0}$ is the residue corresponding to eigenvalue 0 and $\|R(t)\| \leq C e^{-\varepsilon t}$ for suitable constants $\varepsilon>0$ and $C>0$.

However, by Theorem 19, the nonnegative solution of the system (1)-(3) can be expressed as $P(t, \cdot)=T(t) P_{0}, t \in[0, \infty)$. Then, combing Theorem 12.3 in [36], we can derive that

$$
\begin{aligned}
P(t, \cdot) & =T(t) P_{0}=\left(\bar{P}_{0}+R(t)\right) P_{0} \\
& =\left\langle P_{0}, Q^{*}\right\rangle \widehat{P}+R(t) P_{0}=\widehat{P}+R(t) P_{0},
\end{aligned}
$$

where $Q^{*}$ is defined in Lemma 15 . Hence, we can get

$$
\|P(t, \cdot)-\widehat{P}\| \leq C e^{-\varepsilon t} .
$$

The proof of Theorem 24 is completed.

\section{Reliability Indices}

In this section, we will discuss some reliability indices of the system. Noting that the eigenfunction corresponding to eigenvalue 0 of the system operator $A$ is just the steady-state solution of system (1)-(3), we are dedicated to studying some primary steady-state indices of the system from the point of eigenfunction.

We first analyze the eigenfunction corresponding to eigenvalue 0 of the system operator $A$. In the proof process of Lemma 11, solving (31)-(36) with the help of (37) by letting $\gamma=0$ and $G=0$ derives

$$
P_{1}=\frac{\varepsilon}{\alpha_{0}+\mu_{0}} P_{0}
$$

$$
\begin{gathered}
P_{2}=\frac{\alpha_{0}}{\lambda} P_{0}+\frac{\mu_{0}}{\lambda} P_{3}=\left[\frac{\alpha_{0}}{\lambda}+\frac{\varepsilon \alpha_{0} \mu_{0}}{\lambda\left(\lambda+\mu_{0}\right)\left(\alpha_{0}+\mu_{0}\right)}\right] P_{0}, \\
P_{3}=\frac{\alpha_{0}}{\lambda+\mu_{0}} P_{1}=\frac{\varepsilon \alpha_{0}}{\left(\lambda+\mu_{0}\right)\left(\alpha_{0}+\mu_{0}\right)} P_{0}, \\
P_{4}=\frac{\lambda}{\mu_{0}} P_{3}=\frac{\lambda \varepsilon \alpha_{0}}{\mu_{0}\left(\lambda+\mu_{0}\right)\left(\alpha_{0}+\mu_{0}\right)} P_{0}, \\
P_{5}(x)=\alpha_{0}\left(1+\frac{\varepsilon}{\alpha_{0}+\mu_{0}}\right) P_{0} e^{-\int_{0}^{x} \mu(s) \mathrm{d} s} .
\end{gathered}
$$

Let

$$
P_{5}=\int_{0}^{\infty} P_{5}(x) \mathrm{d} x=\alpha_{0}\left(1+\frac{\varepsilon}{\alpha_{0}+\mu_{0}}\right) P_{0} g(0),
$$


where $g(0)=\int_{0}^{\infty} e^{-\int_{0}^{x} \mu(s) \mathrm{d} s} \mathrm{~d} x$ and

$$
\begin{aligned}
S & =\sum_{i=0}^{5} P_{i} \\
& =\frac{\mu_{0}\left(\lambda+\mu_{0}\right)\left(\alpha_{0}+\mu_{0}+\varepsilon\right)\left(\lambda+\alpha_{0}+\lambda \alpha_{0} g(0)\right)+\lambda^{2} \varepsilon \alpha_{0}}{\lambda \mu_{0}\left(\alpha_{0}+\mu_{0}\right)\left(\lambda+\mu_{0}\right)} P_{0} .
\end{aligned}
$$

Theorem 25. The steady-state availability of the system is

$$
A_{v}=\frac{\mu_{0}\left(\lambda+\alpha_{0}\right)\left(\lambda+\mu_{0}\right)\left(\alpha_{0}+\mu_{0}+\varepsilon\right)}{\mu_{0}\left(\lambda+\mu_{0}\right)\left(\alpha_{0}+\mu_{0}+\varepsilon\right)\left(\lambda+\alpha_{0}+\lambda \alpha_{0} g(0)\right)+\lambda^{2} \varepsilon \alpha_{0}} .
$$

Proof. The instantaneous availability of the system at time $t$ is

$$
A_{v}(t)=\sum_{i=0}^{3} P_{i}(t)
$$

Let $t \rightarrow \infty$, then the steady-state availability of the system is obtained as follows:

$$
\begin{aligned}
A_{v} & =\frac{\sum_{i=0}^{3} P_{i}}{S} \\
& =\frac{\mu_{0}\left(\lambda+\alpha_{0}\right)\left(\lambda+\mu_{0}\right)\left(\alpha_{0}+\mu_{0}+\varepsilon\right)}{\mu_{0}\left(\lambda+\mu_{0}\right)\left(\alpha_{0}+\mu_{0}+\varepsilon\right)\left(\lambda+\alpha_{0}+\lambda \alpha_{0} g(0)\right)+\lambda^{2} \varepsilon \alpha_{0}} .
\end{aligned}
$$

The proof of Theorem 25 is completed.

Theorem 26. The steady-state probability of the repairman vacation is

$$
P_{v}=\frac{\lambda \varepsilon\left(\alpha_{0}+\mu_{0}\right)\left(\lambda+\mu_{0}\right)}{\mu_{0}\left(\lambda+\mu_{0}\right)\left(\alpha_{0}+\mu_{0}+\varepsilon\right)\left(\lambda+\alpha_{0}+\lambda \alpha_{0} g(0)\right)+\lambda^{2} \varepsilon \alpha_{0}} .
$$

Proof. The instantaneous probability of the repairman vacation at time $t$ is

$$
P_{v}(t)=P_{1}(t)+P_{3}(t)+P_{4}(t) .
$$

Letting $t \rightarrow \infty$ derives the steady-state probability of the repairman vacation:

$$
\begin{aligned}
P_{v} & =\frac{P_{1}+P_{3}+P_{4}}{S} \\
& =\frac{\lambda \varepsilon\left(\alpha_{0}+\mu_{0}\right)\left(\lambda+\mu_{0}\right)}{\mu_{0}\left(\lambda+\mu_{0}\right)\left(\alpha_{0}+\mu_{0}+\varepsilon\right)\left(\lambda+\alpha_{0}+\lambda \alpha_{0} g(0)\right)+\lambda^{2} \varepsilon \alpha_{0}} .
\end{aligned}
$$

The proof of Theorem 26 is completed.

Theorem 27. The steady-state probability of the system in warning state is

$$
P_{w}=\frac{\alpha_{0} \mu_{0}\left(\lambda+\mu_{0}\right)\left(\alpha_{0}+\mu_{0}+\varepsilon\right)}{\mu_{0}\left(\lambda+\mu_{0}\right)\left(\alpha_{0}+\mu_{0}+\varepsilon\right)\left(\lambda+\alpha_{0}+\lambda \alpha_{0} g(0)\right)+\lambda^{2} \varepsilon \alpha_{0}} .
$$

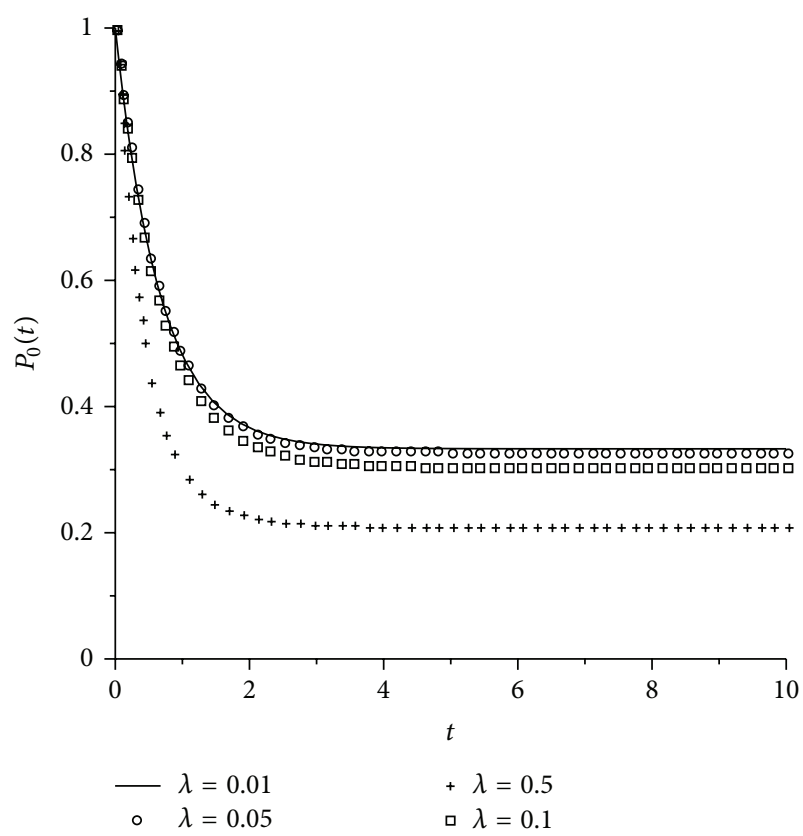

FIGURE 1: Instantaneous probabilities of the system without warning device in good state with different $\lambda$.

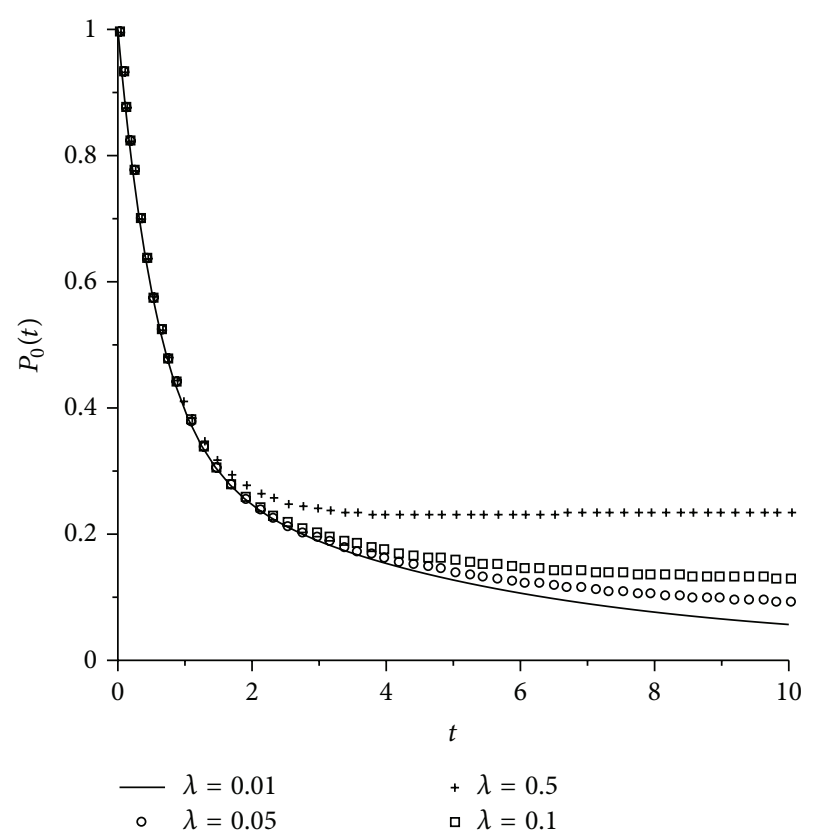

FIGURE 2: Instantaneous probabilities of the system with warning device in good state with different $\lambda$.

Proof. The instantaneous probability of the system in warning state at time $t$ is

$$
P_{w}(t)=P_{2}(t)+P_{3}(t) .
$$




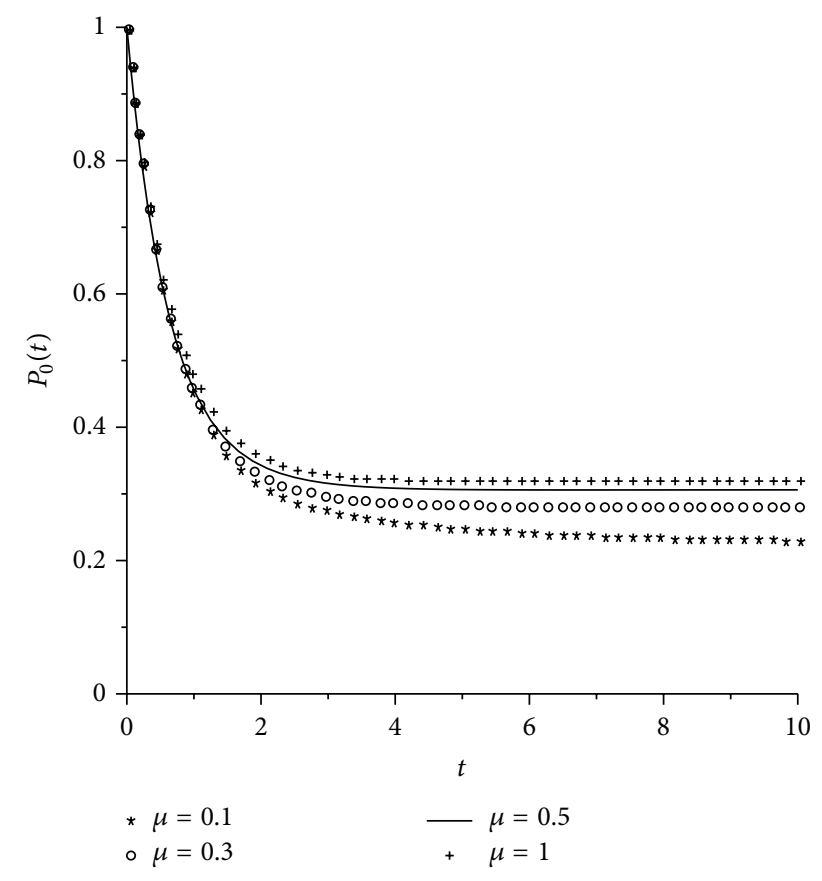

FIGURE 3: Instantaneous probabilities of the system without warning device in good state with different $\mu$.

Letting $t \rightarrow \infty$ yields the steady-state probability of the system in warning state:

$$
\begin{aligned}
P_{w} & =\frac{P_{2}+P_{3}}{S} \\
& =\frac{\alpha_{0} \mu_{0}\left(\lambda+\mu_{0}\right)\left(\alpha_{0}+\mu_{0}+\varepsilon\right)}{\mu_{0}\left(\lambda+\mu_{0}\right)\left(\alpha_{0}+\mu_{0}+\varepsilon\right)\left(\lambda+\alpha_{0}+\lambda \alpha_{0} g(0)\right)+\lambda^{2} \varepsilon \alpha_{0}} .
\end{aligned}
$$

The proof of Theorem 27 is completed.

Theorem 28. The steady-state failure frequency of the system is

$$
W_{f}=\lambda P_{w}
$$

Proof. Let $P_{5}(t)=\int_{0}^{\infty} P_{5}(t, x) \mathrm{d} x$ and $\mu(t)=$ $\int_{0}^{\infty} \mu(x) P_{5}(t, x) \mathrm{d} x / P_{5}(t)$. Then, the matrix of the transition probability of the system (1)-(3) can be obtained by (1)-(2) as follows:

$$
T=\left(\begin{array}{cccccc}
-\varepsilon-\alpha_{0} & \mu_{0} & 0 & 0 & 0 & \mu(t) \\
\varepsilon & -\alpha_{0}-\mu_{0} & 0 & 0 & 0 & 0 \\
\alpha_{0} & 0 & -\lambda & \mu_{0} & 0 & 0 \\
0 & \alpha_{0} & 0 & -\lambda-\mu_{0} & 0 & 0 \\
0 & 0 & 0 & \lambda & -\mu_{0} & 0 \\
0 & 0 & \lambda & 0 & \mu_{0} & -\mu(t)
\end{array}\right)
$$

Thus, by [37] the instantaneous failure frequency of the system at time $t$ can be derived as

$$
W_{f}(t)=\lambda\left[P_{2}(t)+P_{3}(t)\right] .
$$

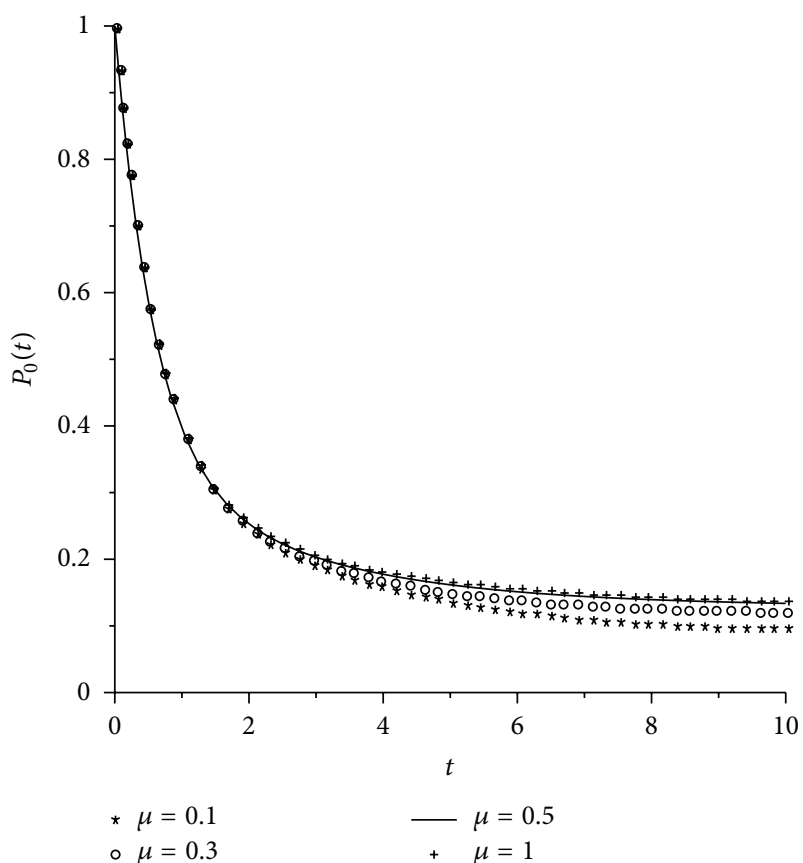

FIgURE 4: Instantaneous probabilities of the system with warning device in good state with different $\mu$.

Let $t \rightarrow \infty$, then the steady-state failure frequency is immediate

$$
W_{f}=\frac{\lambda\left(P_{2}+P_{3}\right)}{S}=\lambda P_{w}
$$

The proof of Theorem 28 is completed.

\section{Applications and Numerical Examples}

Reference [38] discussed the effects of the delayed vacation and vacation policies on a system. That is, the shorter the delayed vacation time, the larger the reliability and failure frequency of a system; and the reliability of a system with multiple vacations is smaller than that of a system with single vacation, while the profit of a system with multiple vacations is larger than that of a system with single vacation. Therefore, in this section, we only concentrate on that how the warning device will affect the system. Specifically, we will compare the reliability, availability, and profit of the system with warning device and those of the system without warning device and present some numerical examples.

6.1. System without Warning Device. The simple repairable system without warning device and with a repairman following delayed-multiple vacations policy is as follows:

$$
\left(\frac{\mathrm{d}}{\mathrm{d} t}+\varepsilon+\lambda\right) P_{0}(t)=\mu_{0} P_{1}(t)+\int_{0}^{\infty} \mu(x) P_{5}(t, x) \mathrm{d} x,
$$

$$
\left(\frac{\mathrm{d}}{\mathrm{d} t}+\mu_{0}+\lambda\right) P_{1}(t)=\varepsilon P_{0}(t)
$$




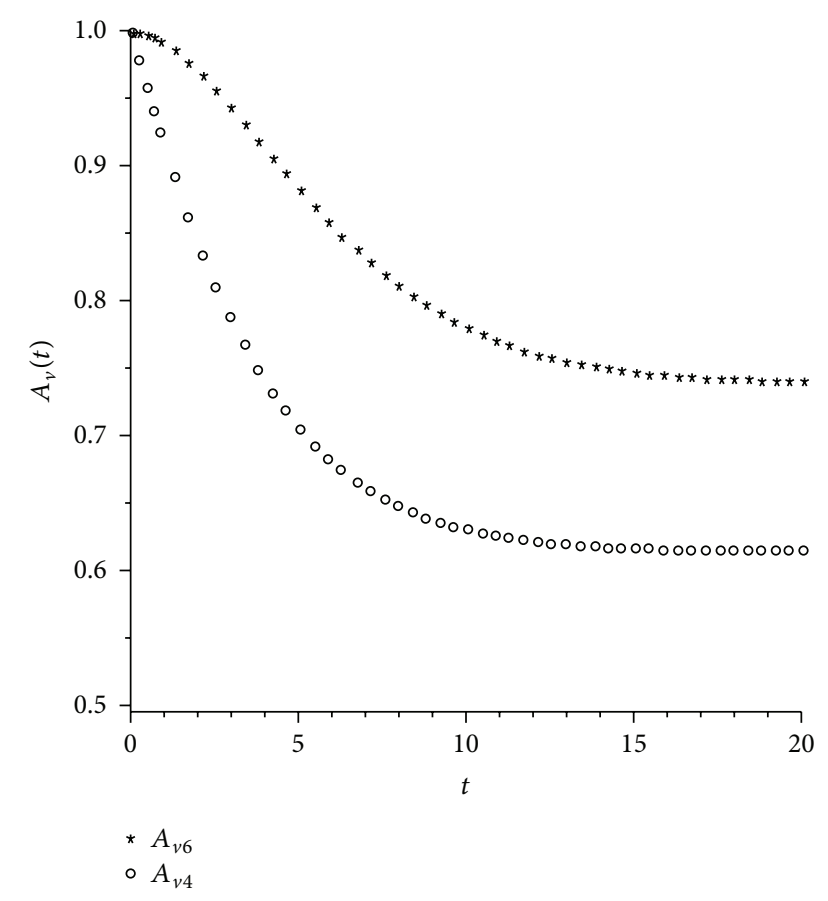

Figure 5: Instantaneous availabilities of the systems with and without warning device.

$$
\begin{gathered}
\left(\frac{\mathrm{d}}{\mathrm{d} t}+\mu_{0}\right) P_{4}(t)=\lambda P_{1}(t) \\
{\left[\frac{\partial}{\partial t}+\frac{\partial}{\partial x}+\mu(x)\right] P_{5}(t, x)=0}
\end{gathered}
$$

with boundary condition

$$
P_{5}(t, 0)=\lambda P_{0}(t)+\mu_{0} P_{4}(t)
$$

and initial conditions

$$
P_{0}(0)=1, \quad \text { the others equal to } 0 \text {. }
$$

Then, by the same method of Theorems 25, 26, and 28, the corresponding reliability indices of the system (133)-(138) are as follows.

Theorem 29. The steady-state availability of the system (133)(138) is

$$
\widetilde{A}_{v}=\frac{\mu_{0}\left(\mu_{0}+\lambda+\varepsilon\right)}{\mu_{0}\left(\mu_{0}+\lambda+\varepsilon\right)(1+\lambda g(0))+\lambda \varepsilon},
$$

where $g(0)$ is defined in Section 5.

Theorem 30. The steady-state probability of the repairman vacation of the system (133)-(138) is

$$
\widetilde{P}_{v}=\frac{\varepsilon\left(\mu_{0}+\lambda\right)}{\mu_{0}\left(\mu_{0}+\lambda+\varepsilon\right)(1+\lambda g(0))+\lambda \varepsilon} .
$$

Theorem 31. The steady-state failure frequency of the system (133)-(138) is

$$
\widetilde{W}_{f}=\lambda A_{v}
$$

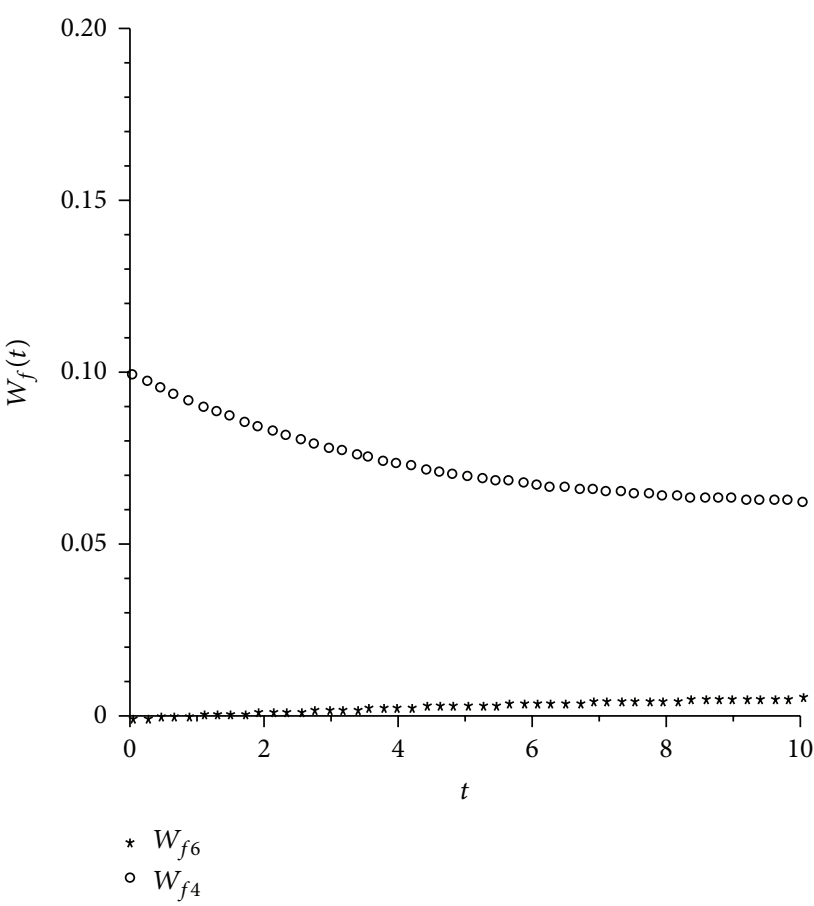

FIGURE 6: Instantaneous failure frequencies of the systems with and without warning device with $\alpha=0.01$.

6.2. Numerical Examples. By comparing the two groups of (120) and (139) and (129) and (141), it is not difficult to deduce the following results.

(i) The steady-state availability of the system with warning device (i.e., system (1)-(3)) is larger than that of the system without warning device (i.e., system (133)(138)). That is, $A_{v}>\widetilde{A}_{v}$.

(ii) If $\alpha_{0} \leq \lambda$, then the steady-state failure frequency of the system with warning device is less than that of the system without warning device. That is, $W_{f}<\widetilde{W}_{f}$. While if $\alpha_{0}>\lambda$, the magnitude of $W_{f}$ and $\widetilde{W}_{f}$ cannot be determined.

Let $I$ and $\widetilde{I}$ be the total profit of the system with and without warning device, respectively. That is,

$$
\begin{aligned}
& I=c_{1} A_{v}-c_{2} W_{f}+c_{3} P_{v}, \\
& \widetilde{I}=c_{1} \widetilde{A}_{v}-c_{2} \widetilde{W}_{f}+c_{3} \widetilde{P}_{v} .
\end{aligned}
$$

Here, $c_{1}, c_{2}$, and $c_{3}$ represent the income of the system for working unit per unit time, the loss of the system for failed unit per unit time, and the income of the system for the repairman vacation per unit time, respectively. Given $\varepsilon=1$, $\mu_{0}=0.5, \alpha_{0}=0.2, c_{1}=50, c_{2}=15, c_{3}=30$, let $D=I-\widetilde{I}$. Then, $D$ is a function of $\lambda$ and $\mu$. From Figure 10, we can know that the profit of the system with warning device is larger than that of the system without warning device.

From the above discussions, we can deduce that because both the availability and profit of a system with warning device are larger than those of a system without warning 




FIGURE 7: Instantaneous failure frequencies of the systems with and without warning device with $\alpha=0.2$.

device. Then, a system with warning device is better than a system without warning device in practice.

In the following, we will present some numerical examples to illustrate the conclusions.

(1) Let $\varepsilon=1, \mu_{0}=\mu=0.5, \alpha_{0}=0.2, \lambda=$ $0.01,0.05,0.1$, and 0.5 , respectively. Figures 1 and 2 present the instantaneous probabilities of the systems with and without warning device in good state with different values of $\lambda$. Let $\varepsilon=1, \lambda=0.1, \mu_{0}=0.5, \alpha_{0}=0.2, \mu=0.1,0.3,0.5$, and 1, respectively. Figures 3 and 4 present the instantaneous probabilities of the systems with and without warning device in good state with different values of $\mu$. From the four figures, we can see that the instantaneous probabilities of the system without warning device in good state decrease with the increasing of $\lambda$, while the instantaneous probabilities of the system with warning device in good state increase with the increasing of $\lambda$. But both the instantaneous probabilities of the systems with and without warning device in good state increase with the increasing of $\mu$ in general.

(2) Let $\varepsilon=1, \lambda=0.1, \mu_{0}=0.5, \mu=0.2, \alpha_{0}=0.2$. Figure 5 presents the instantaneous availabilities of the systems with and without warning device. From the figure, we can see that the availability of the system with warning device is larger than that of the system without warning device.

(3) Let $\varepsilon=1, \lambda=0.1, \mu_{0}=0.5, \mu=0.2$ and $\alpha_{0}=0.01,0.2,1$, and 5 , respectively. Figures $6,7,8$, and 9 present the instantaneous failure frequencies of the systems with and without warning device. It can be derived from the four figures that when $\alpha_{0}<\lambda$, the instantaneous failure frequency of the system with warning device is less than that of the system without warning device. But when $\alpha_{0} \geq$

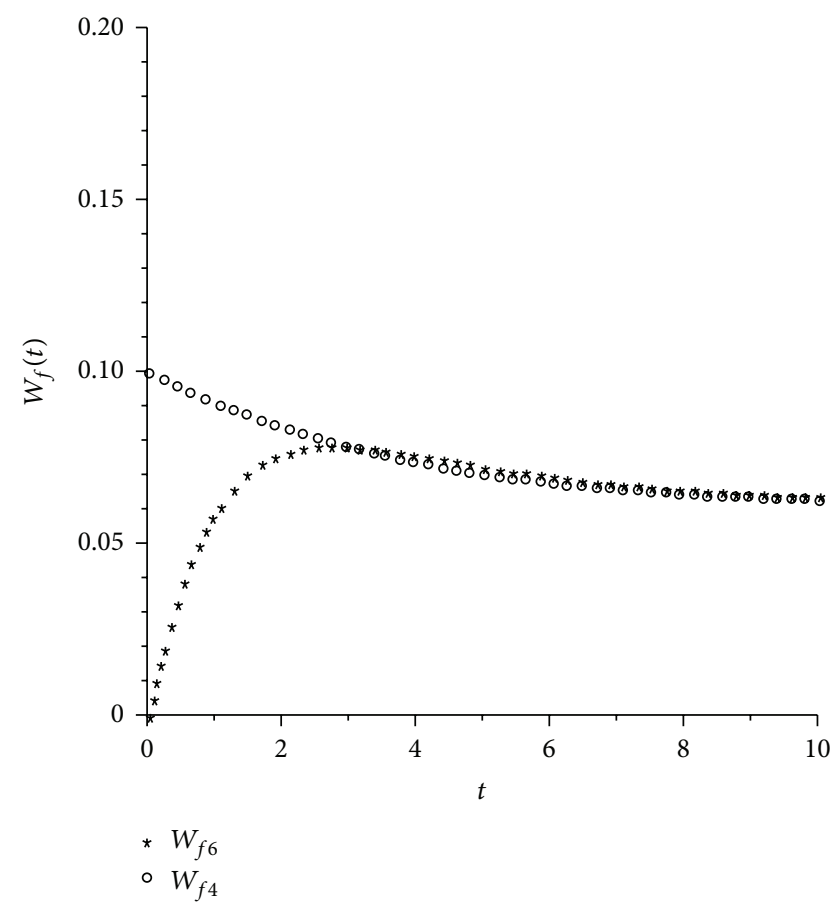

FIGURE 8: Instantaneous failure frequencies of the systems with and without warning device with $\alpha=1$.

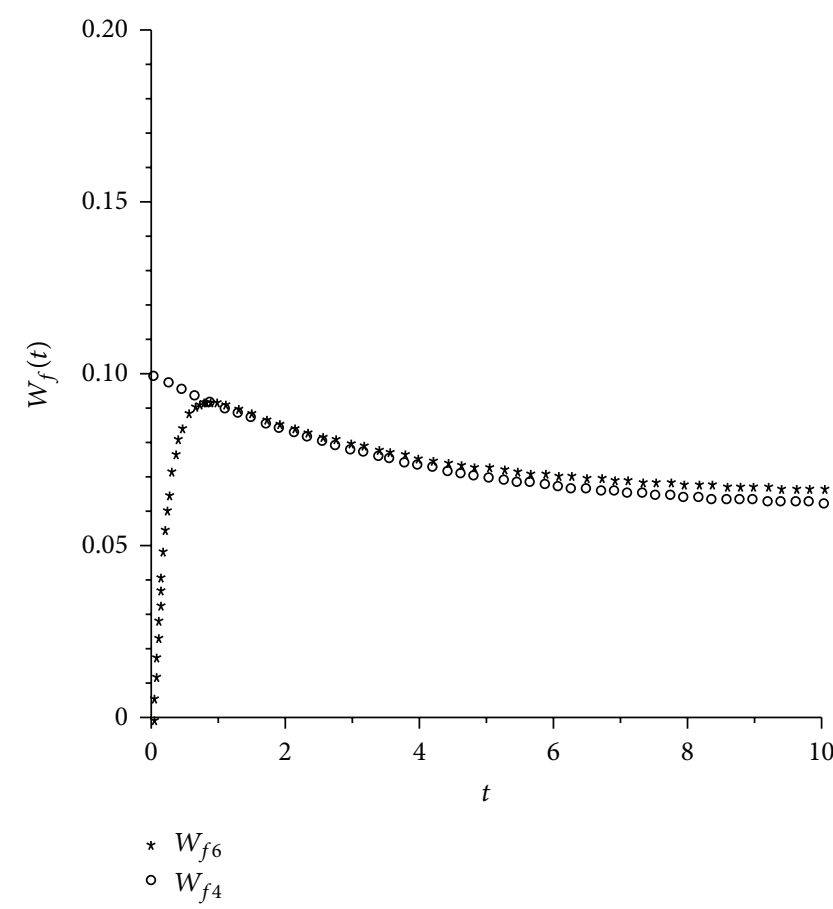

FIGURE 9: Instantaneous failure frequencies of the systems with and without warning device with $\alpha=5$.

$1 \gg \lambda$, the instantaneous failure frequency of the system with warning device is larger than that of the system without warning device. 


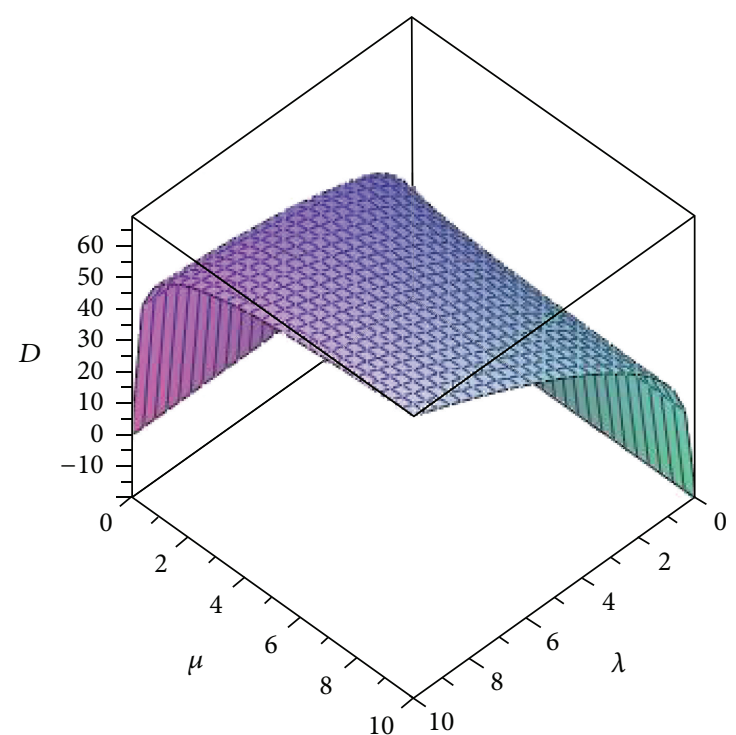

FIGURE 10: Profit difference between the systems with and without warning device.

(4) Let $\varepsilon=1, \mu_{0}=0.5, \alpha_{0}=0.2, c_{1}=50, c_{2}=15, c_{3}=30$. Figure 10 presents the profit difference between the systems with and without warning device. From the figure, it can be derived easily that the profit the system with warning device is more than that of the system without warning device.

\section{Conclusion}

In this paper, we proposed a simple repairable system with a warning device and a repairman who can have delayedmultiple vacations. Because the two hypotheses used for Laplace transform in order to obtain the steady-state solution of a repairable system in traditional reliability research that needs to be verified, and the substitution of steady-state solution for the dynamic one that should be based on some conditions, the study of well-posedness of the time-dependent solution of a system is in demand in terms of theory and practice. In this paper, we first transformed the system model into a group of operator equations and obtained the existence and uniqueness as well as $C^{1}$ continuity of the system solution by functional analysis method. Then to study the stability of the system, we translated the system model into an abstract Cauchy problem in a suitable Banach space. The asymptotic stability and further the exponential stability of the system solution were derived by using $C_{0}$ semigroup theory and compact operator disturbance theorem. Because the stable solution of the system is just the eigenfunction corresponding to eigenvalue 0 of the system operator, we also presented some reliability indices, such as reliability, failure frequency, probabilities of repairman vacation, and system in warning state of the system in the viewpoint of eigenfunction. At the end of the paper, by the theoretical and numerical analyses, we give the conclusion that the system with warning device is better than the system without warning device in practice.

\section{Acknowledgments}

This work is supported by the TianYuan Special Funds of the National Natural Science Foundation of China under Grant no. 11226249, the Foundation Research Project of Shanxi Province (The Youth) under Grant no. 2012021015-5, the Youth Foundation of Taiyuan University of Technology under Grant no. 2012L031, the Special Funds of the National Natural Science Foundation of China under Grant no. 61250011, and the Foundation Research Project of Shanxi Province (The Natural Science) under Grant no. 2012011004-4.

\section{References}

[1] M. Yaez, F. Joglar, and M. Modarres, "Generalized renewal process for analysis of repairable systems with limited failure experience," Reliability Engineering and System Safety, vol. 77, no. 2, pp. 167-180, 2002.

[2] R. K. Mobley, An Introduction to Predictive Maintenance, Elsevier Science, New York, NY, USA, 2nd edition, 2002.

[3] B. T. Doshi, "Queueing systems with vacations-a survey," Queueing Systems, vol. 1, no. 1, pp. 29-66, 1986.

[4] H. Takagi, Queueing Analysis: A Foundation of Performance Evaluation, Vacation and Priority Systems, Part I, vol. 1, NorthHolland, Amsterdam, The Netherlands, 1991.

[5] N. Tian and Z. G. Zhang, Vacation Queueing Models: Theory and Applications, International Series in Operations Research \& Management Science, Springer, New York, NY, USA, 2006.

[6] J. Ke, "Batch arrival queues under vacation policies with server breakdowns and startup/closedown times," Applied Mathematical Modelling, vol. 31, no. 7, pp. 1282-1292, 2007.

[7] B. K. Kumar, R. Rukmani, and V. Thangaraj, "An M/M/C retrial queueing system with Bernoulli vacations," Journal of Systems Science and Systems Engineering, vol. 18, no. 2, pp. 222-242, 2009.

[8] Z. G. Zhang and N. Tian, "Discrete time Geo/G/1 queue with multiple adaptive vacations," Queueing Systems, vol. 38, no. 4, pp. 419-429, 2001.

[9] Z. G. Zhang, "On the three threshold policy in the multi-server queueing system with vacations," Queueing Systems, vol. 51, no. 1-2, pp. 173-186, 2005.

[10] J. Wu, Z. Liu, and Y. Peng, "On the BMAP/G/1 G-queues with second optional service and multiple vacations," Applied Mathematical Modelling. Simulation and Computation for Engineering and Environmental Systems, vol. 33, no. 12, pp. 4314-4325, 2009.

[11] J. C. Ke, C. U. Wu, and Z. G. Zhang, "Recent developments in vacation models: a short survey," International Journal of Operations Research, vol. 7, no. 4, pp. 3-8, 2010.

[12] M. Jain and M. S. Rakhee, "Bilevel control of degraded machining system with warm standbys, setup and vacation," Applied Mathematical Modelling, vol. 28, no. 12, pp. 1015-1026, 2004.

[13] L. M. Hu, D. Q. Yue, and J. D. Li, "Probabilistic analysis of a series-parallel repairable system with three units and vacation," Applied Mathematical Modelling, vol. 34, no. 10, pp. 2711-2721, 2010.

[14] Q. T. Wu and S. M. Wu, "Reliability analysis of two-unit cold standby repairable systems under Poisson shocks," Applied Mathematics and Computation, vol. 218, no. 1, pp. 171-182, 2011. 
[15] L. Yuan, "Reliability analysis for a $k$-out-of- $n$ : $G$ system with redundant dependency and repairmen having multiple vacations," Applied Mathematics and Computation, vol. 218, no. 24, pp. 11959-11969, 2012.

[16] L. Yuan and Z. D. Cui, "Reliability analysis for the consecutive$k$-out-of- $n: F$ system with repairmen taking multiple vacations," Applied Mathematical Modelling, vol. 37, no. 7, pp. 4685-4697, 2013.

[17] W. Z. Yuan and G. Q. Xu, "Modelling of a deteriorating system with repair satisfying general distribution," Applied Mathematics and Computation, vol. 218, no. 11, pp. 6340-6350, 2012.

[18] J. C. Ke and C. H. Wu, "Multi-server machine repair model with standbys and synchronous multiple vacation," Computers and Industrial Engineering, vol. 62, no. 1, pp. 296-305, 2012.

[19] J. C. Ke and K. H. Wang, "Vacation policies for machine repair problem with two type spares," Applied Mathematical Modelling, vol. 31, no. 5, pp. 880-894, 2007.

[20] J. S. Jia and S. M. Wu, "A replacement policy for a repairable system with its repairman having multiple vacations," Computers and Industrial Engineering, vol. 57, no. 1, pp. 156-160, 2009.

[21] L. Yuan and J. Xu, "A deteriorating system with its repairman having multiple vacations," Applied Mathematics and Computation, vol. 217, no. 10, pp. 4980-4989, 2011.

[22] L. Yuan and J. Xu, "An optimal replacement policy for a repairable system based on its repairman having vacations," Reliability Engineering and System Safety, vol. 96, no. 7, pp. 868875, 2011.

[23] M. M. Yu, Y. H. Tang, L. P. Liu, and J. Cheng, "A phase-type geometric process repair model with spare device procurement and repairman's multiple vacations," European Journal of Operational Research, vol. 225, no. 2, pp. 310-323, 2013.

[24] L. N. Guo, H. B. Xu, C. Gao, and G. T. Zhu, "Stability analysis of a new kind series system," IMA Journal of Applied Mathematics, vol. 75, no. 3, pp. 439-460, 2010.

[25] L. N. Guo, H. B. Xu, C. Gao, and G. T. Zhu, "Stability analysis of a new kind $n$-unit series repairable system," Applied Mathematical Modelling, vol. 35, no. 1, pp. 202-217, 2011.

[26] M. E. Gurtin and R. C. MacCamy, "Non-linear age-dependent population dynamics," Archive for Rational Mechanics and Analysis, vol. 54, pp. 281-300, 1974.

[27] Y. Yao, "Global existence and uniqueness of solutions to epidemic dynamic systems of equations," Chinese Annals of Mathematics A, vol. 12, no. 2, pp. 218-230, 1991.

[28] P. Yan, "Well-posedness of a model for the dynamics of infectious diseases without immunity and with latent period," Journal of Biomathematics, vol. 23, no. 2, pp. 245-256, 2008.

[29] K. J. Engel and R. Nagel, One-Parameter Semigroups for Linear Evolution Equations, vol. 194 of Graduate Texts in Mathematics, Springer, New York, NY, USA, 2000.

[30] H. B. Xu, W. H. Guo, J. Y. Yu, and G. T. Zhu, "The asymptotic stability of a series repairable system," Acta Mathematicae Applicatae Sinica, vol. 29, no. 1, pp. 46-52, 2006.

[31] N. Dunford and J. T. Schwartz, Linear Operators, Part I, Wiley Classics Library, John Wiley \& Sons, New York, NY, USA, 1958.

[32] A. Pazy, Semigroups of Linear Operators and Applications to Partial Differential Equations, vol. 44 of Applied Mathematical Sciences, Springer, New York, NY, USA, 1983.

[33] G. Geni, X. Z. Li, and G. T. Zhu, Functional Analysis Method in Queueing Theory, Research Information Limited, Hertfordshire, UK, 2001.
[34] P. Clément, H. J. A. M. Heijmans, S. Angenent, C. J. van Duijn, and B. de Pagter, One-Parameter Semigroup, Elsevier Science, Amsterdam, The Netherlands, 1987.

[35] R. Nagel, One-Parameter Semigroups of Positive Operators, vol. 1184 of Lecture Notes in Mathematics, Springer, New York, NY, USA, 1986.

[36] A. E. Taylor and D. C. Lay, Introduction to Functional Analysis, John Wiley \& Sons, New York, NY, USA, 1980.

[37] J. H. Cao and K. Cheng, Introduction to Reliability Mathematics, Higher Education Press, Beijing, China, 1986.

[38] L. N. Guo, Z. X. Zhao, H. B. Xu, and G. T. Zhu, "Reliability and profit analysis of a series repairable system with repairman vacation," in Proceedings of the 30th Chinese Control Conference (CCC '11), pp. 1009-1014, July 2011. 




Advances in

Operations Research

mansans

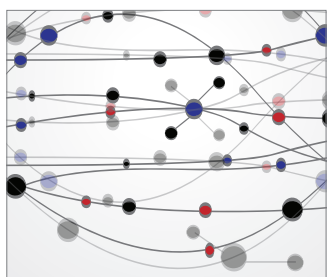

The Scientific World Journal
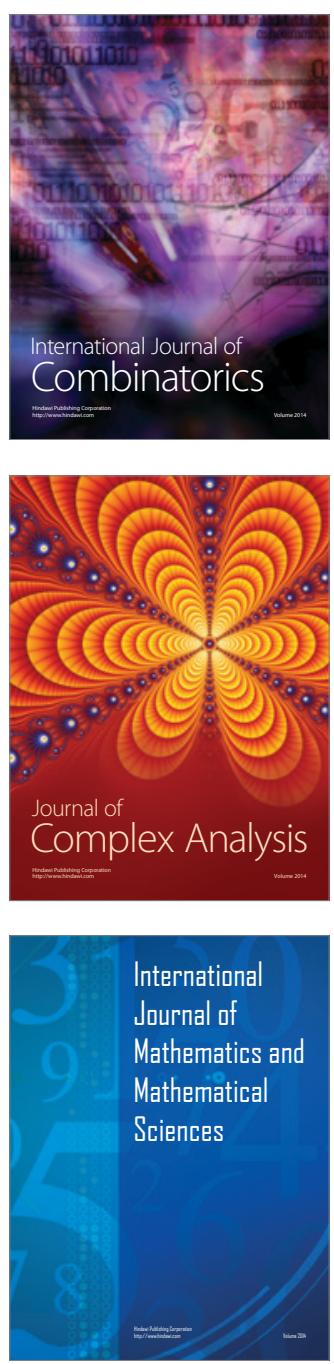
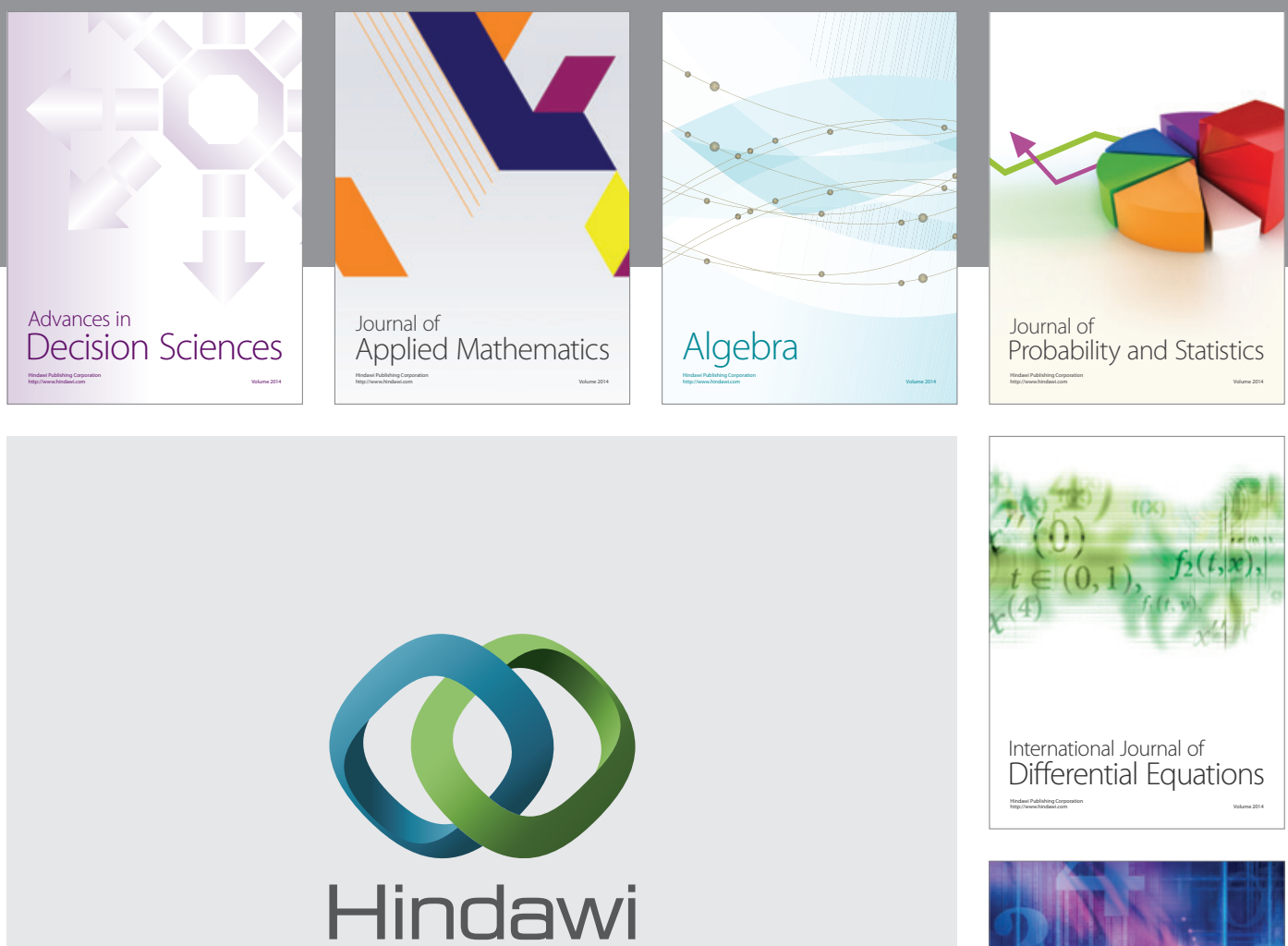

Submit your manuscripts at http://www.hindawi.com
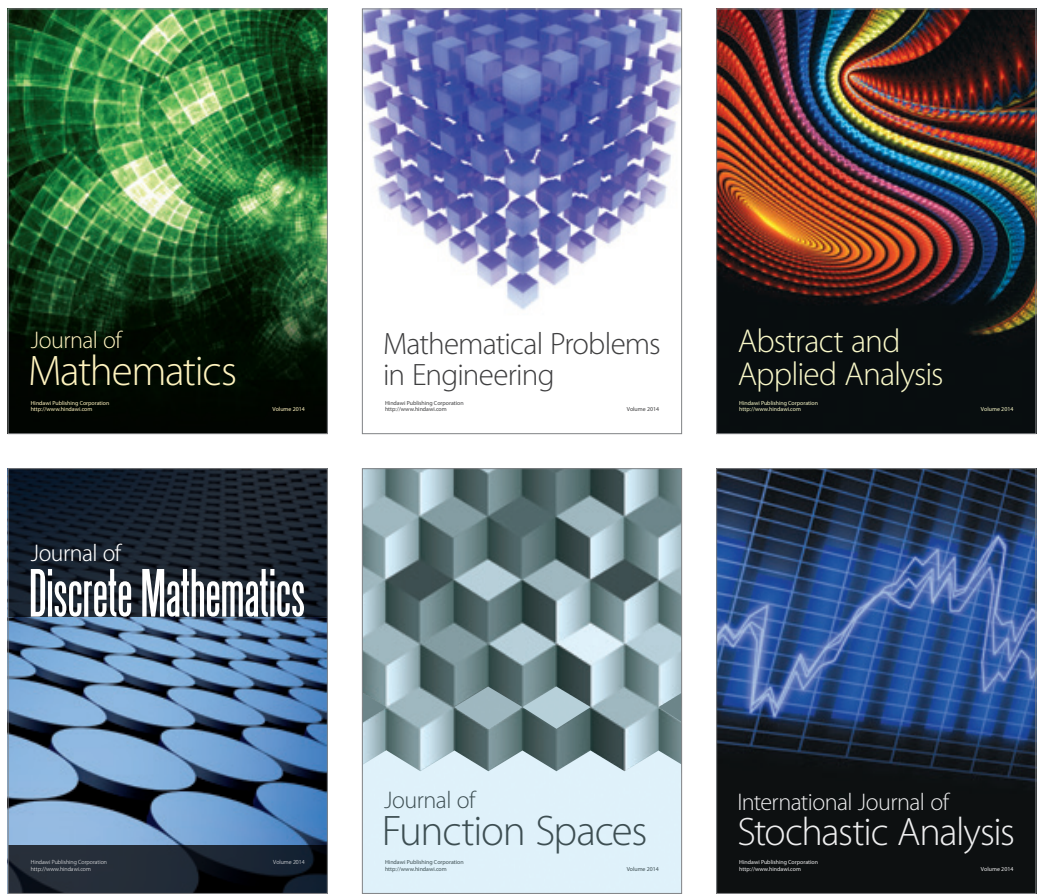

Journal of

Function Spaces

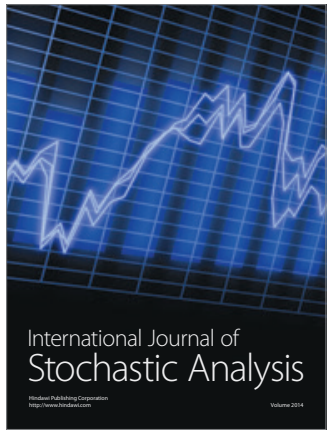

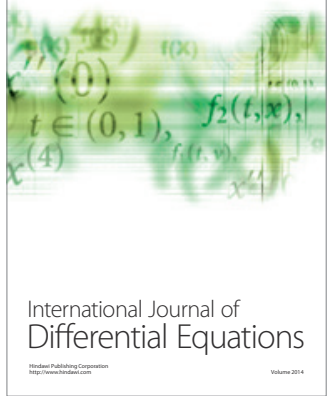
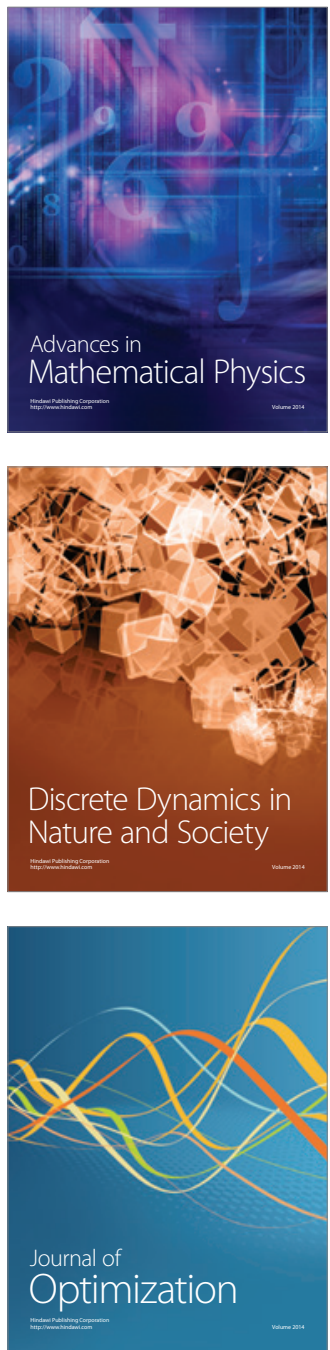